Homology, Homotopy and Applications, vol.11(2), 2009, pp.381-404

\title{
A RELATIVE VERSION OF THE FINITENESS OBSTRUCTION THEORY OF C. T. C. WALL
}

\author{
ANNA DAVIS
}

\author{
(communicated by Charles A. Weibel)
}

\begin{abstract}
In his 1965 paper C. T. C. Wall demonstrated that if a CW complex $Y$ is finitely dominated, then the reduced projective class group of $Y$ contains an obstruction which vanishes if and only if $Y$ is homotopy equivalent to a finite $\mathrm{CW}$ complex. Wall also demonstrated that such an obstruction is invariant under homotopy equivalences. Subsequently Sum and Product Theorems for this obstruction were proved by L. C. Siebenmann.

In his second paper on the subject Wall gives an algebraic definition of the relative finiteness obstruction. If a CW complex $Y$ is finitely dominated rel. a subcomplex $X$, then the reduced projective class group of $Y$ contains an obstruction which vanishes if and only if $Y$ is homotopy equivalent to a finite complex rel. $X$.

In this paper we will use a geometric construction to reduce the relative finiteness obstruction to the non-relative version. We will demonstrate that the relative finiteness obstruction is invariant under certain types of homotopy equivalences. We will also prove the relative versions of the Sum and the Product Theorems.
\end{abstract}

\section{Introduction}

The purpose of this paper is to provide a geometric reduction of Wall's relative finiteness obstruction [14] to his non-relative version [13]. The idea that such a reduction is possible as well as the choice of construction techniques used in this paper are due to Chapman. We will employ a modification of the infinite mapping cylinder construction used by Ferry in [7] to obtain a geometric description of the non-relative obstruction. Our modification is similar to the technique used by Chapman in [5] and involves a truncated version of the infinite mapping cylinder construction. As a result we obtain relative versions of the sum and product theorems of [10].

We will start by reviewing the non-relative theory. In what follows all spaces are assumed to be locally compact, separable and metric and all maps are continuous functions.

Received August 26, 2006, revised September 9, 2009; published on December 2, 2009.

2000 Mathematics Subject Classification: 57Q12.

Key words and phrases: CW complex, finiteness obstruction, relative finiteness obstruction.

This article is available at http://intlpress.com/HHA/v11/n2/a17

Copyright (C) 2009, International Press. Permission to copy for private use granted. 
A map $d: X \longrightarrow Y$ is a homotopy domination if there exists a map $u: Y \longrightarrow X$ such that $d u \simeq i d_{Y}$. We say that $u$ is an inverse of $d$ and that $Y$ is dominated by $X$. A CW complex is said to be finitely dominated if it is dominated by a finite complex.

Wall's finiteness obstruction theory arises from the question: If $Y$ is finitely dominated, when is $Y$ homotopy equivalent to some finite $C W$ complex? Wall defines the finiteness obstruction of a finitely dominated CW complex $Y$ to be an element $\sigma(Y)$ of the reduced projective class group $\tilde{K}_{0}\left(Z \pi_{1}(Y)\right)$ and shows that $\sigma(Y)=0$ if and only if $Y$ is homotopy equivalent to a finite complex [13]. Wall also shows that $\sigma(Y)$ is an invariant of homotopy type. Siebenmann used finiteness obstruction to solve the problem of putting a boundary on an open manifold [10]. He also obtained product and sum theorems for the obstruction $[\mathbf{1 0}]$.

By a $C W$ pair $(Y, X)$ we mean a $C W$ complex $Y$ together with a subcomplex $X$ closed in $Y$. Let $(Y, X)$ be a $\mathrm{CW}$ pair. $Y$ is said to be finitely dominated rel. $X$ if there exists a compact CW complex $K$ and a map $d: X \cup K \rightarrow Y$ such that $d$ is a homotopy domination rel. $X$ (i.e., there exists a homotopy inverse $u$ such that $d u \stackrel{h_{t}}{\simeq} i d$ with $h_{t}(x)=x$ for all $\left.x \in X\right)$. Equivalently, $Y$ is finitely dominated rel. $X$ if the inclusion $i: X \cup C \hookrightarrow Y$ is a homotopy domination rel. $X$ for some compact subcomplex $C \subset Y$. If $Y$ is finitely dominated rel. $X$ we say that $(Y, X)$ is finitely dominated.

Let $\left(Y_{1}, X\right)$ and $\left(Y_{2}, X\right)$ be CW pairs. Suppose there is a homotopy equivalence $f: Y_{1} \rightarrow Y_{2}$ such that $\left.f\right|_{X}: X \rightarrow X$ is the identity. If there exists an inverse $g$ such that $\left.g\right|_{X}: X \rightarrow X$ is the identity, and homotopies $F_{t}$ and $G_{t}$ such that $f g \stackrel{F_{t}}{\simeq} i d, g f \stackrel{G_{t}}{\simeq} i d$ and $\left.F_{t}\right|_{X}=i d,\left.G_{t}\right|_{X}=i d$ for all $t$, then we say that $f$ is a homotopy equivalence rel. $X$. If $Y_{2}=X \cup K$, where $K$ is a finite complex, we say that $Y_{1}$ is homotopy equivalent rel. $X$ to a finite complex.

Relative finiteness obstruction theory arises from the question: If $Y$ is finitely dominated rel. $X$, when is $Y$ homotopy equivalent to a finite complex rel. $X$ ? Wall uses relative chain complexes to define the relative finiteness obstruction of a finitely dominated pair $(Y, X)$ to be an element $\sigma(Y, X)$ of $\tilde{K}_{0}\left(Z \pi_{1}(Y)\right)$ [14]. The obstruction vanishes if and only if $Y$ is homotopy equivalent rel. $X$ to some $X \cup K$, where $K$ is finite. The relative finiteness obstruction plays a key role in infinite simple homotopy theory $[\mathbf{1 1}]$.

We will reduce the relative finiteness obstruction to the ordinary finiteness obstruction and derive the relative versions of the sum and product formulas. The ideas for the sum and product formulas came from Cohen who proves similar results for Whitehead torsion in [6]. Statements of Theorem 4.15 and Lemma 4.17 are analogous to Cohen's 20.2 and 20.3. The striking similarity between formulas and results for the relative finiteness obstruction and Whitehead torsion can be partially explained by the Bass-Heller-Swan result which states that the reduced projective class group of $Y$ injects into the Whitehead group of $Y \times S^{1}[\mathbf{1}]$.

\section{Acknowledgements}

This paper is based on my doctoral dissertation completed under the direction of Thomas Chapman, University of Kentucky. I would like to thank Thomas Chapman for his guidance and support. 


\section{Preliminaries}

\subsection{Finiteness Obstruction}

Let $X$ be a connected complex. Let $Z \pi_{1}(X)=Z \pi$ denote the integral group ring. The reduced projective class group of $X, \tilde{K}_{0}\left(Z \pi_{1}(X)\right)$, can be thought of as the Grothendieck group of finitely generated projective $Z \pi$-modules, modulo free modules [13]. Henceforth we will refer to $\tilde{K}_{0}\left(Z \pi_{1}(X)\right)$ as $\tilde{K}_{0}(X)$. The reduced projective class group of a non-connected complex $X$ is the direct sum of the reduced projective class groups of the components of $X[\mathbf{1 0}]$.

$\tilde{K}_{0}$ is a functor from the category of topological spaces and continuous functions to the category of abelian groups and homomorphisms [10]. $\tilde{K}_{0}$ satisfies the following properties:

1. For any space $X, \tilde{K}_{0}(X)$ is an abelian group.

2. If $f: X \rightarrow Y$ is a continuous function then there is an induced homomorphism $f_{*}: \tilde{K}_{0}(X) \rightarrow \tilde{K}_{0}(Y)$.

3. $i d_{*}=i d$ and $(g f)_{*}=g_{*} f_{*}$.

4. $\tilde{K}_{0}$ is a homotopy functor i.e., $f \simeq g$ then $f_{*}=g_{*}$.

5. $\tilde{K}_{0}$ has compact support i.e.,

(a) If $\sigma \in \tilde{K}_{0}(X)$ then there exists a compact $C \subset X$ and $\tau \in \tilde{K}_{0}(C)$ such that $i_{*}(\tau)=\sigma$, where $i: C \hookrightarrow X$ is an inclusion.

(b) If $C \subset X$ is compact, $\tau \in \tilde{K}_{0}(C)$, and $i_{*}(\tau)=0 \in \tilde{K}_{0}(X)$ then there exists a compact $D \subset X$ such that $C \subset D$ and $j_{*}(\tau)=0 \in \tilde{K}_{0}(D)$, where $j: C \hookrightarrow D$ is an inclusion.

If $X$ is a CW complex we can ensure that $C$ and $D$ are finite subcomplexes.

6. Let $X, X^{\prime}, Y$ and $Y^{\prime}$ be finitely dominated spaces. Let $f: X \rightarrow X^{\prime}$ and $g: Y \rightarrow$ $Y^{\prime}$ be maps. There exists a pairing $\cdot: \tilde{K}_{0}(X) \times \tilde{K}_{0}(Y) \longrightarrow \tilde{K}_{0}(X \times Y)$. This pairing is natural in the sense that the following diagram commutes.

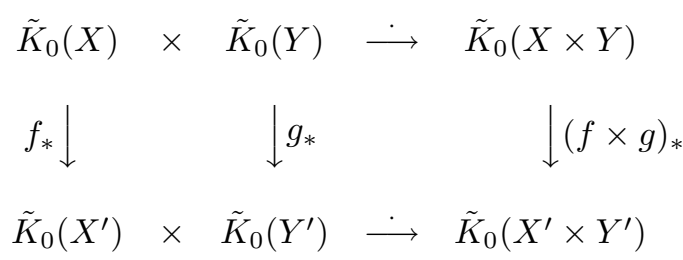

Let $d: K \rightarrow X$ be a map. We will use $\pi_{n}(d)$ to denote $\pi_{n}(M(d), K \times 1)$, where $M(d)$ is the mapping cylinder of $d$. We say that $d$ is n-connected if $K$ and $X$ are connected and $\pi_{i}(d)=0$ for $1 \leqslant i \leqslant n$.

Definition $2.1([\mathbf{1 3}]) . \quad 1$. We say that $X$ satisfies $F_{1}$ if $\pi$ is finitely generated.

2. We say that $X$ satisfies $F_{2}$ if $\pi$ is finitely presented, and for any finite complex $K^{2}$ and map $\phi: K^{2} \rightarrow X$ inducing an isomorphism of fundamental groups, $\pi_{2}(\phi)$ is a finitely generated module over $Z \pi$.

3. We say that $X$ satisfies $F_{n}(n \geqslant 3)$ if it satisfies $F_{n-1}$ and for any finite complex $K^{n-1}$ and $(n-1)$-connected map $\phi: K^{n-1} \rightarrow X, \pi_{n}(\phi)$ is a finitely generated $Z \pi$-module. 
Definition $2.2([\mathbf{1 3}])$. We say that $X$ satisfies $D_{n}$ if $H_{i}(\tilde{X})=0$ for $i>n$, and $H^{n+1}(X ; \mathbf{B})=0$ for all coefficient bundles $\mathbf{B}$.

The following is a statement of Wall's theorem of [13] as it appears in [12].

Theorem 2.3. $X$ is dominated by a finite complex if and only if $X$ satisfies $F_{N}$ and $D_{N}$ for some $N \geqslant 3$. In this case $X$ satisfies $F_{n}$ and $D_{n}$ for all $n \geqslant N$. Moreover, given any $n \geqslant N$ we can find an $n$-connected map $D: L=L^{n} \rightarrow X$ with $L$ finite. In this case $\pi_{n+1}(D)$ is finitely generated, projective over $Z \pi$ and the element $\sigma(X)=(-1)^{n+1}\left[\pi_{n+1}(D)\right] \in \tilde{K}_{0}(X)$ depends only on the homotopy type of $X$. The vanishing of $\sigma(X)$ is necessary and sufficient for $X$ to be homotopy equivalent to a finite complex.

In the above theorem, $D$ is an extension of a finite domination $d: K \rightarrow X$, and $L$ is obtained by attaching a finite number of cells to $K[\mathbf{1 3}]$.

The finiteness obstruction is defined to be $\sigma(X)=(-1)^{n+1}\left[\pi_{n+1}(D)\right]$. Obstruction may also be defined geometrically as in [7].

The finiteness obstruction satisfies the following properties:

1. Invariance (Wall [13])

If $f: X \rightarrow Y$ is a homotopy equivalence and both spaces are finitely dominated, then $f_{*}(\sigma(X))=\sigma(Y)$.

2. Sum Theorem (Siebenmann [10])

If $X=X_{1} \cup X_{2}$, where $X_{1}, X_{2}, X_{0}=X_{1} \cap X_{2}$ are finitely dominated, then $X$ is finitely dominated and

$$
\sigma(X)=j_{1 *} \sigma\left(X_{1}\right)+j_{2 *} \sigma\left(X_{2}\right)-j_{0 *} \sigma\left(X_{0}\right)
$$

where $j_{i}: X_{j} \hookrightarrow X$ are inclusions.

3. Product Theorem (Siebenmann $[\mathbf{1 0}]$ )

If $X_{1}$ and $X_{2}$ are finitely dominated, connected CW complexes then $X_{1} \times X_{2}$ is finitely dominated and

$$
\sigma\left(X_{1} \times X_{2}\right)=\sigma\left(X_{1}\right) \cdot \sigma\left(X_{2}\right)+\chi\left(X_{2}\right) j_{1 *} \sigma\left(X_{1}\right)+\chi\left(X_{1}\right) j_{2 *} \sigma\left(X_{2}\right),
$$

where $j_{i}: X_{i} \hookrightarrow X_{1} \times X_{2}$ are inclusions, and $\chi$ is the Euler Characteristic function.

\subsection{Relative Finiteness Obstruction}

We will reduce the relative finiteness obstruction to the ordinary obstruction as follows. Given a finitely dominated pair $(Y, X)$ we construct a CW pair $\left(X \cup D^{\prime}, X\right)$ and a homotopy equivalence $u: X \cup D^{\prime} \rightarrow Y$ rel. $X$, where $D^{\prime}$ is finitely dominated and $u\left(D^{\prime}\right)$ is contained in a compact subset of $Y$. Then $\sigma(Y, X)$ is defined to be the image of $\sigma\left(D^{\prime}\right)$ in $\tilde{K}_{0}\left(Z \pi_{1}(Y)\right)$. This definition allows us to naturally deduce the relative versions of the sum and product theorems from the non-relative versions of $[\mathbf{1 0}]$.

1. Fundamental Property of Relative Finiteness Obstruction $\sigma(Y, X)=0$ if and only if $Y$ is homotopy equivalent rel. $X$ to a finite complex. 
2. Relative Invariance

If $(Y, X)$ is finitely dominated and $f:(Y, X) \rightarrow\left(Y_{1}, X_{1}\right)$ is a homotopy equivalence such that $\left.f\right|_{X}: X \rightarrow X_{1}$ is a homotopy equivalence, then $\left(Y_{1}, X_{1}\right)$ is finitely dominated and $f_{*}(\sigma(Y, X))=\sigma\left(Y_{1}, X_{1}\right)$.

3. Relative Sum Theorem

Suppose $Y=Y_{1} \cup Y_{2}, Y_{0}=Y_{1} \cap Y_{2}$ and $X=X_{1} \cup X_{2}, X_{0}=X_{1} \cap X_{2}$, where $X_{1} \subset Y_{1}, X_{2} \subset Y_{2}$, and $X_{0} \subset Y_{0}$, and each $\left(Y_{i}, X_{i}\right)$ is finitely dominated $(i=$ $0,1,2)$. Then $(Y, X)$ is finitely dominated and

$$
\sigma(Y, X)=j_{1 *}\left(\sigma\left(Y_{1}, X_{1}\right)\right)+j_{2 *}\left(\sigma\left(Y_{2}, X_{2}\right)\right)-j_{0 *}\left(\sigma\left(Y_{0}, X_{0}\right)\right)
$$

where $j_{i}: Y_{i} \hookrightarrow Y$ are inclusions.

4. Relative Product Theorem

If $\left(Y_{1}, X_{1}\right)$ and $\left(Y_{2}, X_{2}\right)$ are finitely dominated, path connected CW pairs, then $\left(Y_{1} \times Y_{2},\left(Y_{1} \times X_{2}\right) \cup\left(X_{1} \times Y_{2}\right)\right)$ is finitely dominated and

$$
\begin{gathered}
\sigma\left(Y_{1} \times Y_{2},\left(Y_{1} \times X_{2}\right) \cup\left(X_{1} \times Y_{2}\right)\right)=\sigma\left(Y_{1}, X_{1}\right) \cdot \sigma\left(Y_{2}, X_{2}\right)+ \\
\chi\left(Y_{1}, X_{1}\right) j_{2 *} \sigma\left(Y_{2}, X_{2}\right)+\chi\left(Y_{2}, X_{2}\right) j_{1 *} \sigma\left(Y_{1}, X_{1}\right),
\end{gathered}
$$

where $j_{i}: Y_{i} \hookrightarrow Y_{1} \times Y_{2}$ are inclusions.

\subsection{Miscellaneous}

A $C W$ isomorphism between two CW complexes $Y_{1}$ and $Y_{2}$ is a homeomorphism of $Y_{1}$ onto $Y_{2}$ such that the image of every cell of $Y_{1}$ is a cell of $Y_{2}$. A map is cellular provided that it sends every cell into cell(s) of equal or lower dimension.

A direct mapping cylinder $D(f)$ of a map $f: X \rightarrow X$ is the space formed from the disjoint union of $X \times[n, n+1]$, where $n$ is an integer, by identifying $(x, n) \in$ $X \times[n-1, n]$ with $(f(x), n) \in X \times[n, n+1]$ for each $x \in X$.

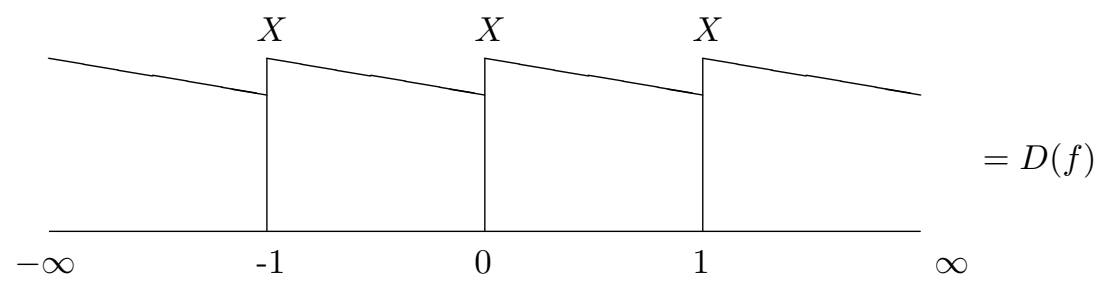

The Hilbert Cube is the countable infinite product

$$
Q=\Pi_{i=1}^{\infty} I_{i},
$$

where each $I_{i}=[-1,1]$. Every separable metric space can be embedded into $Q$. Let $Q_{0}$ denote the Hilbert Cube with one point removed. It is easy to show that if a space $Z$ is an ANR, then there exists a proper embedding $k: Z \rightarrow Q_{0}$. (Let $\tilde{Z}$ be a one-point compactification of $Z$. Embed $\tilde{Z}$ into $Q$. Remove the image of $\infty$ from $Q$.) For more information on the Hilbert Cube, see [4]. 


\section{Preliminary Constructions}

Definition 3.1. Let $(Y, X)$ be a finitely dominated CW pair. Let $D=D^{\prime} \cup X$, where $D^{\prime}$ is a finitely dominated subcomplex of $D$. If there exists a homotopy equivalence $\hat{u}: D \rightarrow Y$ rel. $X$ with homotopy inverse $\hat{v}$ such that $\hat{u}\left(D^{\prime}\right)$ is contained in a compact set, then we say that $(Y, X)$ is stable with respect to $\left(D^{\prime}, \hat{u}, \hat{v}\right)$. When inverse $\hat{v}$ of $\hat{u}$ is irrelevant, we will say that $(Y, X)$ is stable with respect to $\left(D^{\prime}, \hat{u}\right)$ or simply, stable.

The construction that we carry out will result in $\hat{u}\left(D^{\prime}\right)$ being compact.

This section is devoted to the proof of the following theorem.

Theorem 3.2. Every finitely dominated $C W$ pair is stable.

Let $C \subset Y$ be a finite subcomplex such that $X \cup C \hookrightarrow Y$ is a homotopy domination rel. $X$ (i.e., $\exists$ a homotopy $h_{t}: Y \rightarrow Y$ rel. $X$ such that $h_{0}=i d$ and $h_{1}(Y) \subset$ $X \cup C)$. Let $e=\left.h_{1}\right|_{X \cup C}: X \cup C \rightarrow X \cup C$. Observe that $e$ is the identity on $X$, so $e$ is cellular on $X$. By $[\mathbf{9}] e$ is homotopic rel. $X$ to a cellular map. Thus we can assume that $e$ is cellular. Form the direct mapping cylinder $D(e)$. By 3.5 of $[6] D(e)$ is a CW complex.

For the purpose of Lemma 3.3 and definitions of $u$ and $v$ we will refer to a point of the direct mapping cylinder $D(e)$ as $(x, n+t)$, where $x \in X \cup C, 0 \leqslant t<1$ and $n$ is an integer. Subsequently we will refer to points of $D(e)$ as $(x, t)$, where $t \in R$.

Define maps

$$
\begin{gathered}
u: D(e) \longrightarrow Y, \quad u(x, n+t)=h_{t}(x) \\
v: Y \longrightarrow D(e), \quad v(y)=\left(h_{1}(y), 0\right) .
\end{gathered}
$$

Lemma 3.3. $u v \simeq i d_{Y}$ and $v u \simeq i d_{D(e)}$.

Proof. Observe that

$$
u v=h_{1} \simeq i d .
$$

Define $T: D(e) \longrightarrow D(e)$ by

$$
T(x, n+t)=(x, n+t-1) .
$$

We will first show that $v u \simeq T$.

Define $\tilde{u}: D(e) \longrightarrow Y \times R$ and $\tilde{v}: Y \times R \longrightarrow D(e)$ by

$$
\begin{aligned}
& \tilde{u}(x, n+t)= \begin{cases}(x, n+2 t) & 0 \leqslant t \leqslant \frac{1}{2} \\
\left(h_{2 t-1}(x), n+1\right) & \frac{1}{2} \leqslant t<1\end{cases} \\
& \tilde{v}(y, n+t)= \begin{cases}\left(h_{1}(y), n+2 t\right) & 0 \leqslant t<\frac{1}{2} \\
\left(h_{1} h_{2-2 t}(y), n+1\right) & \frac{1}{2} \leqslant t<1 .\end{cases}
\end{aligned}
$$

We want to show that $\tilde{v} \tilde{u} \simeq T$. 
Define a homotopy $\psi_{s}: D(e) \longrightarrow D(e)$ by

$$
\psi_{s}(x, n+t)= \begin{cases}\left(h_{1}(x), n+4 t\right) & 0 \leqslant t<\frac{1}{4} \\ \left(h_{1} h_{2-4 t+s(4 t-1)}(x), n+1\right) & \frac{1}{4} \leqslant t \leqslant \frac{1}{2} \\ \left(h_{1} h_{2 t-1+s(2-2 t)}(x), n+1\right) & \frac{1}{2} \leqslant t<1 .\end{cases}
$$

Then

$$
\begin{gathered}
\psi_{0}=\tilde{v} \tilde{u} \\
\psi_{1}(x, n+t)= \begin{cases}\left(h_{1}(x), n+4 t\right) & 0 \leqslant t<\frac{1}{4} \\
\left(h_{1} h_{1}(x), n+1\right) & \frac{1}{4} \leqslant t<1 .\end{cases}
\end{gathered}
$$

Let

$$
\theta(x, n+t)=\left(h_{1}(x), n+t\right) .
$$

Then $\psi_{1} \simeq \theta$ via the homotopy

$$
H_{s}^{3}(x, n+t)= \begin{cases}\left(h_{1}(x), n+t+3 t s\right) & 0 \leqslant t \leqslant \frac{1}{4} \\ r_{s}\left(h_{1}(x), n+t\right) & \frac{1}{4} \leqslant t<1\end{cases}
$$

where

$$
r_{s}(x, n+t)= \begin{cases}(x, n+t+s(1-t)) & 0 \leqslant s<1 \\ \left(h_{1}(x), n+1\right) & s=1 .\end{cases}
$$

Now we need to show that $T \simeq \theta$. Define $G_{s}: D(e) \rightarrow D(e)$ by

$$
G_{s}(x, n+t)= \begin{cases}(x, n+t+s) & 0 \leqslant s<1-t \\ \left(h_{1}(x), n+t+s\right) & 1-t \leqslant s \leqslant 1 .\end{cases}
$$

Observe that

$$
\begin{gathered}
G_{0}(x, n+t)=(x, n+t)=i d(x, n+t) \\
G_{1}(x, n+t)=\left(h_{1}(x), n+t+1\right)=\theta T^{-1}(x, n+t) .
\end{gathered}
$$

Assuming that $G$ is continuous we have $i d \simeq \theta T^{-1}$. Therefore $T \simeq \theta$.

We now need to show that $G$ is continuous. We will demonstrate that $G$ is continuous on a closed segment of the direct mapping cylinder. Continuity of $G$ will follow from the Pasting Lemma.

Let $M_{1}$ be the mapping cylinder of $e: X \cup C \rightarrow e(X \cup C)$. Let $M_{2}$ be the mapping cylinder of $\left.e\right|_{e(X \cup C)}: e(X \cup C) \rightarrow e(e(X \cup C))$. Let $M=M_{1} \cup M_{2}$ (1-level of $M_{1}$ is identified with the 0 -level of $M_{2}$ ).

Consider the following diagram:

$$
\begin{aligned}
& ((X \cup C) \times[0,1]) \times I \quad \stackrel{F}{\longrightarrow} \quad(X \cup C) \times[0,2] \\
& \downarrow q=q_{1} \times i d \quad \downarrow q_{2} \\
& M_{1} \times I \quad \stackrel{\left.G\right|_{M_{1} \times I}}{\longrightarrow} \quad M
\end{aligned}
$$


where the maps are defined as follows

$$
\begin{aligned}
F(x, n+t, s) & =(x, n+t+s) \\
q_{1}(x, n+t) & = \begin{cases}(x, n+t) & n=0 \\
\left(h_{1}(x), 1\right) & n=1\end{cases} \\
q_{2}(x, n+t) & = \begin{cases}(x, n+t) & n=0 \\
\left(h_{1}(x), n+t\right) & n=1 \\
\left(h_{1} h_{1}(x), 2\right) & n=2 .\end{cases}
\end{aligned}
$$

Observe that the diagram commutes. Since $q$ is a proper continuous surjection it follows that $q$ is a quotient map.

To show continuity of $G$ pick an open set $U \subset M$, then

$$
q^{-1}\left(G^{-1}(U)\right)=F^{-1}\left(q_{2}^{-1}(U)\right)=\left(q_{2} F\right)^{-1}(U)
$$

but $\left(q_{2} F\right)^{-1}(U)$ is open since $q_{2} F$ is continuous. Therefore $q^{-1}\left(G^{-1}(U)\right)$ is open. But then $G^{-1}(U)$ is open because $q$ is a quotient map. So $G$ is continuous.

Let $p=$ proj: $Y \times R \longrightarrow Y$. Define $i: Y \hookrightarrow Y \times R$ by $i(y)=(y, 0)$. Let $u^{\prime}(x$, $n+t)=\left(h_{t}(x), n+t\right)$.

We have the following sequence of homotopies:

$$
v u=\tilde{v} i u=\tilde{v} i p u^{\prime} \stackrel{H_{s}^{1}}{\simeq} \tilde{v} i p \tilde{u} \stackrel{H_{s}^{2}}{\simeq} \tilde{v} \tilde{u}=\psi_{0} \stackrel{\psi_{s}}{\simeq} \psi_{1} \stackrel{H_{s}^{3}}{\simeq} \theta \stackrel{G_{s}}{\simeq} T
$$

where

$$
\begin{aligned}
& H_{s}^{1}(x, n+t)= \begin{cases}\tilde{v}\left(h_{t s}(x), 0\right) & 0 \leqslant t \leqslant \frac{1}{2} \\
\tilde{v}\left(h_{(2 t-1)(1-s)+s t}(x), 0\right) & \frac{1}{2} \leqslant t<1\end{cases} \\
& H_{s}^{2}(x, n+t)= \begin{cases}\tilde{v}(x, n+2 t(1-s)) & 0 \leqslant t \leqslant \frac{1}{2} \\
\tilde{v}\left(h_{(2 t-1)}(x), n+(1-s)\right) & \frac{1}{2} \leqslant t<1 .\end{cases}
\end{aligned}
$$

Finally we need to show that $T \simeq i d_{D(e)}$. Observe that $T$ is a homeomorphism. Thus $T=T^{-1} T T \simeq T^{-1} v(u v) u \simeq T^{-1} v u \simeq T^{-1} T=i d$.

Lemma 3.4. There exists a compact subcomplex $V$ of $X \cup C$ such that $C \subset \operatorname{Int}(V)$ and $h_{1} h_{t_{1}} h_{t_{2}}(C) \subset \operatorname{Int}(V)$ for all $t_{1}, t_{2} \in I$.

Proof. Recall that $h: Y \times I \rightarrow Y$ is a homotopy. Then $h(C \times I) \subset A$ for some compact $A \subset Y$. Thus $h_{t_{2}}(C) \subset A$ for all $t_{2} \in I$.

Also, $h(A \times I) \subset B$ for some compact $B \subset Y$. Thus $h_{t_{1}}(A) \subset B$ for all $t_{1} \in I$. Therefore there exists a compact subcomplex $V$ such that $C \subset \operatorname{Int}(V)$ and

$$
h_{1} h_{t_{1}} h_{t_{2}}(C) \subset h_{1} h_{t_{1}}(A) \subset h_{1}(B) \subset \operatorname{Int}(V) \subset X \cup C .
$$

\section{Constructing $D^{\prime}$}

Choose a subcomplex $V$ of $X \cup C$ as in Lemma 3.4. Let $D^{\prime \prime}$ be the direct mapping cylinder of $\left.e\right|_{V}$. Then $D^{\prime \prime} \subset D(e)$.

Let $X^{\prime}$ be a finite subcomplex of $X \cup C$ containing $V$ in its interior. Let $D^{\prime}$ be the direct mapping cylinder of $e^{\prime}=\left.e\right|_{X^{\prime}}$. Then $D^{\prime} \subset D(e)$. Since $e^{\prime}$ is cellular, $D^{\prime}$ is 
a subcomplex of $D=X \cup D^{\prime}$, where the union is taken by identifying $X^{\prime} \subset X$ with $X^{\prime} \times\{0\} \subset D^{\prime}$. Note that since $v u(x, t)=v\left(h_{t}(x)\right)=\left(h_{1} h_{t}(x), 0\right)$, we have $v u\left(D^{\prime}\right) \subset$ $X^{\prime} \times\{0\}$. Thus, $D^{\prime}$ is finitely dominated.

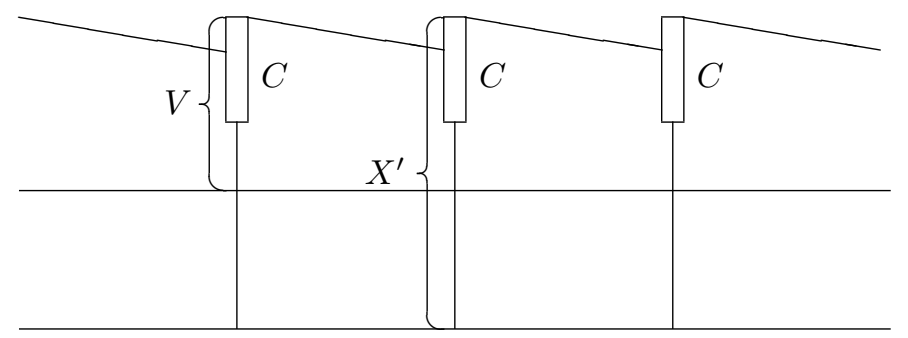

Figure 1: $D^{\prime}$

Lemma 3.5. The homotopy $v u \simeq i d_{D(e)}$ restricts to $\left.v u\right|_{D^{\prime}} \simeq i d_{D^{\prime}}$.

Proof. If we use homotopies of Lemma 3.3 together with Lemma 3.4 it is easy to check that the path of each element of $D^{\prime}$ lies in $D^{\prime}$.

Theorem 3.6. There exists a homotopy equivalence $\hat{u}: D^{\prime} \cup X \rightarrow Y$ such that $\hat{u}\left(D^{\prime}\right)$ is compact.

Proof. We will treat $D(e)$ as $D^{\prime \prime} \cup((X \backslash V) \times R)$.

Choose a map $\phi: X^{\prime} \backslash \operatorname{Int}(V) \rightarrow[0,1]$ such that $\phi\left(B d\left(X^{\prime}\right)\right)=1$ and $\phi(B d(V))=0$. Define $r: D(e) \rightarrow D(e)$ by

$$
r(x, t)= \begin{cases}(x, t) & x \in V \\ (x, 0) & x \in X \backslash X^{\prime} \\ \left(x, \frac{1}{\phi(x)}-1\right) & x \in X^{\prime} \backslash V, t>\frac{1}{\phi(x)}-1 \\ \left(x,-\frac{1}{\phi(x)}+1\right) & x \in X^{\prime} \backslash V, t<-\frac{1}{\phi(x)}+1 \\ (x, t) & x \in X^{\prime} \backslash V,|t| \leqslant \frac{1}{\phi(x)}-1 .\end{cases}
$$

Clearly $r$ is a strong deformation retraction, i.e., there exists a map (a horizontal push)

$$
g_{s}: D(e) \longrightarrow D(e)
$$

such that $g_{0}=i d, g_{1}(D(e))=r(D(e))$ and $\left.g_{t}\right|_{r(D(e))}=i d$. (See Figure 2) Thus,

$$
X \cup D^{\prime} \stackrel{k}{\longleftrightarrow} D(e)
$$

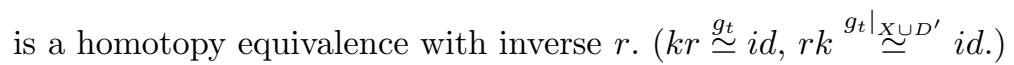

Let

$$
\hat{u}=u k=\left.u\right|_{X \cup D^{\prime}}
$$

then

$$
\hat{u}: X \cup D^{\prime} \longrightarrow Y
$$

is a homotopy equivalence with a homotopy inverse $v^{\prime}=r v$. 


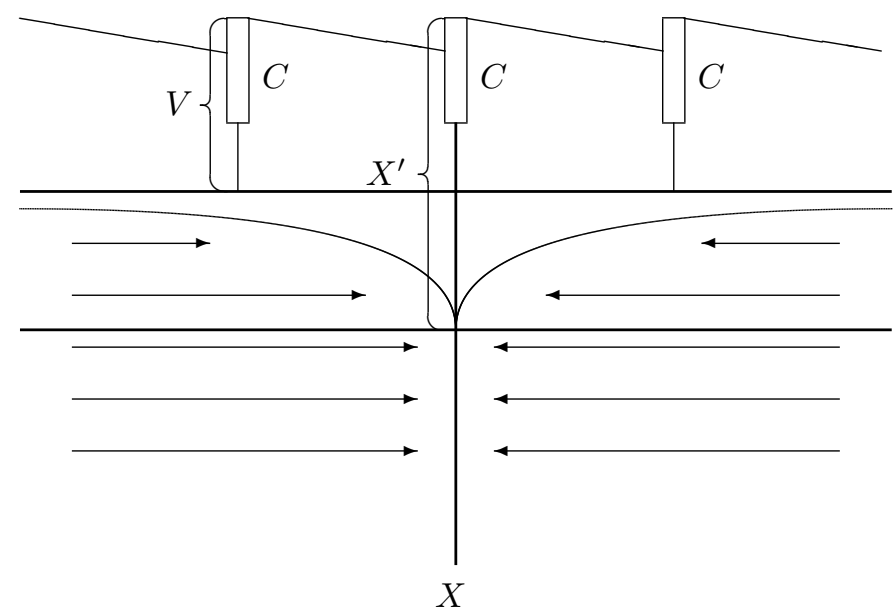

Figure 2: Action of $g_{s}$ on $D(e)$.

Each "cell" $X^{\prime} \times I$ of the direct mapping cylinder $D^{\prime}$ is compact. Therefore $\hat{u}\left(X^{\prime} \times I\right)$ is compact. Because of the way $u$ is defined, the images of all these "cells" coincide in $Y$. Thus, $\hat{u}\left(D^{\prime}\right)$ is compact in $Y$.

To complete the proof of Theorem 3.2 we need to show that $\hat{u}$ is a bounded homotopy equivalence rel. $X$.

Theorem 3.7. Let $(Y, X)$ be a $C W$ pair. Let $Z$ be a locally compact ANR containing $X^{\prime}$ as a closed subset. Suppose $f: Z \rightarrow Y$ is a homotopy equivalence such that $\left.f\right|_{X^{\prime}}: X^{\prime} \rightarrow X$ is a homeomorphism. Then there exists a homotopy inverse $g$ such that $\left.g\right|_{X}: X \rightarrow X^{\prime}$ is a homeomorphism and $g f \simeq i d_{Z}$ rel. $X^{\prime}$ and $f g \simeq i d_{Y}$ rel. $X$.

Proof. Let $k: Z \rightarrow Q_{0}$ be a proper embedding. Define $f^{\prime}: Z \rightarrow Y \times Q_{0}$ by

$$
f^{\prime}(z)=(f(z), k(z)) .
$$

It can be shown that $f^{\prime}$ is a proper embedding. Thus $f^{\prime}(Z)$ is closed in $Y \times Q_{0}$.

There exists some homotopy inverse $g^{\prime}$ of $f$. Define $r^{\prime}: Y \times Q_{0} \rightarrow f^{\prime}(Z) \subset Y \times Q_{0}$ by

$$
r^{\prime}=f^{\prime} g^{\prime} \pi_{Y}
$$

where $\pi_{Y}$ is a projection map in the $Y$ coordinate. Let $i: f^{\prime}(Z) \hookrightarrow Y \times Q_{0}$ be an inclusion. Since $i r^{\prime}(y, t)=\left(f g^{\prime}(y), k g^{\prime}(y)\right)$ and $f g^{\prime} \simeq i d$ we have $i r^{\prime} \simeq i d$.

Let $G$ be a homotopy such that $G_{0}=i d$ and $G_{1}=g^{\prime} f$. Let $F_{t}=\left.f^{\prime} G_{t}\left(f^{\prime}\right)^{-1}\right|_{f^{\prime}(Z)}$ then for any $(f(z), k(z)) \in f^{\prime}(Z)$ we have:

$$
\begin{gathered}
F_{0}(f(z), k(z))=\left.f^{\prime} G_{0}\left(f^{\prime}\right)^{-1}\right|_{f^{\prime}(Z)}(f(z), k(z))=f^{\prime} G_{0}(z)=f^{\prime}(z)=(f(z), k(z)) \\
F_{1}(f(z), k(z))=f^{\prime} G_{1}(z)=f^{\prime} g^{\prime} f(z)=f^{\prime} g^{\prime} \pi_{Y}(f(z), k(z)) \\
=\left.r^{\prime}\right|_{f^{\prime}(Z)}(f(z), k(z))=r^{\prime} i(f(z), k(z)) .
\end{gathered}
$$


Therefore $r^{\prime} i \simeq i d$.

$f^{\prime}(Z)$ is closed in $Y \times Q_{0}$ therefore, by the Homotopy Extension Theorem, there exists a homotopy $H:\left(Y \times Q_{0}\right) \times I \rightarrow Y \times Q_{0}$ such that $H_{1}=r^{\prime}$ and $\left.H_{t}\right|_{f^{\prime}(Z)}=F_{t}$.

Let

$$
r=H_{0} .
$$

Then $r^{\prime} \simeq r$ and $r i=i d_{f^{\prime}(Z)}$. Thus, $r$ is a retraction homotopic to the identity. Using the proof of Theorem 3.1 of $[\mathbf{3}]$ we can ensure that $r \simeq i d$ rel. $f^{\prime}(Z)$.

Let $w: Y \rightarrow Q_{0}$ be a map which extends $\left.k f\right|_{X} ^{-1}$. Define $j: Y \rightarrow Y \times Q_{0}$ by

$$
j(y)=(y, w(y)) .
$$

Since $Q_{0} \cong Q \times[0,1)$ is convex and $\left.w f\right|_{X^{\prime}}=\left.k\right|_{X^{\prime}}$, there is a straight line homotopy $w f \simeq k$ rel. $X^{\prime} \cdot f^{\prime}(z)=(f(z), k(z))$ and $j f(z)=(f(z), w f(z))$. Since the first coordinates agree and other coordinates are joined by a homotopy rel. $X^{\prime}$ we conclude that $j f \simeq f^{\prime}$ rel. $X^{\prime}$.

Define $g: Y \rightarrow Z$ by

$$
g=\left(f^{\prime}\right)^{-1} r j .
$$

Then for any $x \in X \subset Y$ we have

$$
\begin{aligned}
g(x) & =\left(f^{\prime}\right)^{-1} r j(x) \\
& =\left(f^{\prime}\right)^{-1} r(x, w(x)) \\
& =\left(f^{\prime}\right)^{-1} r\left(f\left(\left.f\right|_{X} ^{-1}(x)\right), k\left(\left.f\right|_{X} ^{-1}(x)\right)\right) \\
& =\left(f^{\prime}\right)^{-1} r f^{\prime}\left(\left.f\right|_{X} ^{-1}(x)\right) \\
& =\left(f^{\prime}\right)^{-1} f^{\prime}\left(\left.f\right|_{X} ^{-1}(x)\right) \\
& =\left.f\right|_{X} ^{-1}(x) .
\end{aligned}
$$

Thus, $\left.g\right|_{X}: X \rightarrow X^{\prime}$ is a homeomorphism.

We will now show that $f g \simeq i d_{Y}$ rel. $X$ and $g f \simeq i d_{Z}$ rel. $X^{\prime}$. rj takes $Y$ to $f^{\prime}(Z)$. Since $f^{\prime}$ is an embedding, every point of $f^{\prime}(Z)$ can be expressed as $(f(z), k(z))$ in terms of some $z \in Z$. But then we have

$$
f\left(f^{\prime}\right)^{-1}(f(z), k(z))=f(z)=\pi_{Y}(f(z), k(z)) ;
$$

therefore,

$$
f g=f\left(f^{\prime}\right)^{-1} r j=\pi_{Y} r j .
$$

$r \simeq i d$ therefore

$$
\pi_{Y} r j \simeq \pi_{Y} j
$$

This homotopy can be chosen to be rel. $X$ because for all $x \in X$ we have

$$
j(x)=(x, w(x))=\left(f\left(\left.f\right|_{X} ^{-1}(x)\right), k\left(\left.f\right|_{X} ^{-1}(x)\right)\right)=f^{\prime}\left(\left.f\right|_{X} ^{-1}(x)\right) \in f^{\prime}(Z)
$$

and the homotopy $r \simeq i d$ is rel. $f^{\prime}(Z)$. But $\pi_{Y} j=i d$; therefore, $f g \simeq i d$ rel. $X$.

Recall that $j f \simeq f^{\prime}$ rel. $X^{\prime}$ and that $\left.r\right|_{f^{\prime}(Z)}=i d$. We have

$$
g f=\left(f^{\prime}\right)^{-1} r j f \simeq\left(f^{\prime}\right)^{-1} r f^{\prime}=\left(f^{\prime}\right)^{-1} f^{\prime}=i d
$$

with the homotopy taking place rel. $X^{\prime}$. 
Let $\hat{v}$ be a homotopy inverse of $\hat{u}$ as in Theorem 3.7. Then $(Y, X)$ is stable with respect to $\left(D^{\prime}, \hat{u}, \hat{v}\right)$. This completes the proof of Theorem 3.2.

\section{Relative Finiteness Obstruction: Definition and Properties}

Definition 4.1. Let $(Y, X)$ be a finitely dominated CW pair stable with respect to $\left(D^{\prime}, u, v\right)$. Then we have

$$
D^{\prime} \stackrel{i}{\longrightarrow} D \stackrel{u}{\longrightarrow} Y
$$

where $i$ is an inclusion map. This induces

$$
\tilde{K}_{0}\left(D^{\prime}\right) \stackrel{i_{*}}{\longrightarrow} \tilde{K}_{0}(D) \stackrel{u_{*}}{\longrightarrow} \tilde{K}_{0}(Y) .
$$

$D^{\prime}$ is finitely dominated, so $\sigma\left(D^{\prime}\right) \in \tilde{K}_{0}\left(D^{\prime}\right)$. Define the relative finiteness obstruction $\sigma(Y, X)$ by

$$
\sigma(Y, X)=u_{*} i_{*} \sigma\left(D^{\prime}\right)
$$

Lemma 4.2. Let $D=D^{\prime} \cup X$ be as in Definition 4.1. Then there exists a map $\alpha: D \rightarrow D$ such that $\alpha \stackrel{F_{t}}{\simeq}$ id rel. $X$ and $F_{t}\left(D^{\prime}\right) \subset D^{\prime}, \alpha(D) \subset X \cup C$, where $C$ is compact in $D^{\prime}$.

Proof. $D^{\prime}$ is finitely dominated, therefore there exists a finite subcomplex $P$ of $D^{\prime}$ and a map $\beta: D^{\prime} \rightarrow P$ such that $i \beta \stackrel{f_{t}}{\simeq} i d\left(f_{0}=i d, f_{1}=i \beta\right)$.

Let $X^{\prime}=D^{\prime} \cap X$. We can modify $\beta=i \beta: D^{\prime} \rightarrow D^{\prime}$ so that $\beta \simeq i d_{D^{\prime}}$ rel. $X^{\prime}$. Choose finite subcomplexes $V$ and $W$ of $D^{\prime}$ so that $X^{\prime} \subset \operatorname{Int}(V) \subset V \subset \operatorname{Int}(W)$. Choose a map $\phi: W \backslash \operatorname{Int}(V) \rightarrow[0,1]$ such that $\phi(B d(W))=1$ and $\phi(B d(V))=0$. Define $\tilde{f}_{t}: D^{\prime} \rightarrow D^{\prime}$ by

$$
\tilde{f}_{t}(x)= \begin{cases}f_{t}(x) & x \in D^{\prime} \backslash \operatorname{Int}(W) \\ x & x \in V \\ f_{\phi(x) t}(x) & x \in W \backslash \operatorname{Int}(V) .\end{cases}
$$

Observe that $\tilde{f}_{1} \simeq i d$ rel. $X^{\prime}$. Extend $\tilde{f}_{1}$ to $\alpha: D \rightarrow D$ by the identity. Then $\alpha \simeq i d$ rel. $X$. Let $C=P \cup W$, then $\alpha(D) \subset X \cup C$.

Lemma 4.3. Let $D_{1}=D_{1}^{\prime} \cup X_{1}, D_{2}=D_{2}^{\prime} \cup X_{2}$ be as in Definition 4.1. Suppose $\gamma: D_{1} \rightarrow D_{2}$ is a homotopy equivalence such that $\left.\gamma\right|_{X_{1}}: X_{1} \rightarrow X_{2}$ is a homeomorphism. Then there exists a homotopy equivalence $f^{\prime}: D_{1} \rightarrow D_{2}$ with inverse $g$ such that

(1) $g f^{\prime} \stackrel{F_{t}}{\simeq} i d_{D_{1}}$ rel. $X_{1}$, and $F_{t}\left(D_{1}^{\prime}\right) \subset D_{1}^{\prime} \cup K_{1}$, where $K_{1}$ is compact in $X_{1}$.

(2) $f^{\prime} g \stackrel{G_{t}}{\simeq} i d_{D_{2}}$ rel. $X_{2}$, and $G_{t}\left(D_{2}^{\prime}\right) \subset D_{2}^{\prime} \cup K_{2}$, where $K_{2}$ is compact in $X_{2}$.

Proof. We will prove (1), the proof of (2) is similar. For $i=1,2$ let $\alpha_{i}: D_{i} \rightarrow D_{i}$ be a homotopy equivalence rel. $X_{i}$ such that $\alpha_{i}\left(D_{i}^{\prime}\right) \subset C_{i}$, where $C_{i}$ is compact in $D_{i}^{\prime}$ as in Lemma 4.2. Let $\lambda_{t}^{i}$ be a homotopy joining each $\alpha_{i}$ with $i d_{D_{i}}\left(\lambda_{0}^{i}=i d, \lambda_{1}^{i}=\alpha_{i}\right)$. 
Let $\tau$ be a homotopy inverse of $\gamma$ as in Theorem 3.7 with $\tau \gamma \stackrel{T_{t}}{\simeq} i d_{D_{1}}$ rel. $X_{1}\left(T_{0}=i d\right.$, $\left.T_{1}=\tau \gamma\right)$ and $\gamma \tau \simeq i d_{D_{2}}$ rel. $X_{2}$. Let

$$
\begin{aligned}
& f^{\prime}=\gamma \alpha_{1} \\
& g=\tau \alpha_{2} .
\end{aligned}
$$

Clearly $g f^{\prime}=\tau \alpha_{2} \gamma \alpha_{1} \simeq i d$ rel. $X_{1}$ and $f^{\prime} g=\gamma \alpha_{1} \tau \alpha_{2} \simeq i d$ rel. $X_{2}$.

Define $F_{t}: D_{1} \rightarrow D_{1}$ by

$$
F_{t}(x)= \begin{cases}\lambda_{3 t}^{1}(x) & 0 \leqslant t \leqslant 1 / 3 \\ T_{3 t-1} \alpha_{1}(x) & 1 / 3 \leqslant t \leqslant 2 / 3 \\ \tau \lambda_{3 t-2}^{2} \gamma \alpha_{1}(x) & 2 / 3 \leqslant t \leqslant 1 .\end{cases}
$$

It is easy to check that $F_{t}$ is continuous and that $F_{0}=i d$ and $F_{1}=\tau \alpha_{2} \gamma \alpha_{1}=g f^{\prime}$.

Now we show that $F_{t}\left(D_{1}^{\prime}\right) \subset D_{1}^{\prime} \cup K_{1}$ for some compact $K_{1} \subset X_{1}$. For $t \in\left[0, \frac{1}{3}\right]$ the homotopy stays in $D_{1}^{\prime}$, as in Lemma 4.2. For $t \in\left[\frac{1}{3}, \frac{2}{3}\right]$ observe that $\alpha_{1}\left(D_{1}^{\prime}\right) \subset C_{1}$. Therefore $T_{t} \alpha_{1}\left(D_{1}^{\prime}\right) \subset T\left(C_{1} \times I\right)$ which is compact. Finally, $\gamma \alpha_{1}\left(D_{1}^{\prime}\right)$ is contained in some compact set $C \subset D_{2}$. Therefore $\lambda_{t}^{2} \gamma \alpha_{1}\left(D_{1}^{\prime}\right) \subset \lambda^{2}(C \times I)$ which is compact in $D_{2}$. Thus, $\tau \lambda_{t}^{2} \gamma \alpha_{1}\left(D_{1}^{\prime}\right) \subset \tau \lambda^{2}(C \times I)$ which is compact in $D_{1}$.

Corollary 4.4. Let $\left(Y_{1}, X_{1}\right)$ and $\left(Y_{2}, X_{2}\right)$ be finitely dominated $C W$ pairs. Suppose $\left(Y_{1}, X_{1}\right)$ is stable with respect to $\left(D_{1}^{\prime}, u_{1}, v_{1}\right)$, and $\left(Y_{2}, X_{2}\right)$ is stable with respect to $\left(D_{2}^{\prime}, u_{2}, v_{2}\right)$, and suppose that there exists a homotopy equivalence $f: Y_{1} \rightarrow Y_{2}$ such that $\left.f\right|_{X_{1}}: X_{1} \rightarrow X_{2}$ is a homeomorphism. Then there exists a homotopy equivalence $f^{\prime}: D_{1} \rightarrow D_{2}$ with homotopy inverse $g$ such that conditions (1) and (2) of Lemma 4.3 are satisfied.

Proof. Let $\gamma=v_{2} f u_{1}$ and apply Lemma 4.3.

Corollary 4.5. Let all spaces and maps be as in Lemma 4.3 and Corollary 4.4. In addition assume that $\left.f\right|_{X_{1}}: X_{1} \rightarrow X_{2}$ is a $C W$ isomorphism. Then there exist a finite subcomplex $P_{1} \subset X_{1}$ and a finite subcomplex $P_{2} \subset X_{2}$ such that $\left.f^{\prime}\right|_{D_{1}^{\prime} \cup P_{1}}: D_{1}^{\prime} \cup P_{1} \rightarrow$ $D_{2}^{\prime} \cup P_{2}$ is a homotopy equivalence with homotopy inverse

$$
\left.g\right|_{D_{2}^{\prime} \cup P_{2}}: D_{2}^{\prime} \cup P_{2} \rightarrow D_{1}^{\prime} \cup P_{1} .
$$

Proof. By Lemma 4.3 and Corollary $4.4 f^{\prime}=v_{2} f u_{1} \alpha_{1}$. Find a compact subcomplex $P_{1}$ of $X_{1}$ such that $K_{1} \subset P_{1}$ and $K_{2} \subset f^{\prime}\left(P_{1}\right)$. Observe that $\left.\alpha_{1}\right|_{X_{1}},\left.u_{1}\right|_{X_{1}}$ and $\left.v_{2}\right|_{X_{2}}$ are identity maps. Thus $f^{\prime}\left(P_{1}\right)$ is a subcomplex. Let $P_{2}=f^{\prime}\left(P_{1}\right)$.

Theorem 4.6. Let all spaces and maps be as in Corollary 4.5. Then

$$
f_{*} u_{1 *} i_{1 *} \sigma\left(D^{\prime}\right)=u_{2 *} i_{2 *} \sigma\left(D_{1}^{\prime}\right)
$$

where $i_{1}, i_{2}$ are inclusions.

Proof. By Corollary 4.5 there exist compact $P_{i} \subset X_{i}, i=1,2$, such that

$$
\left.v_{2} f u_{1} \alpha_{1}\right|_{D_{1}^{\prime} \cup P_{1}}: D_{1}^{\prime} \cup P_{1} \rightarrow D_{2}^{\prime} \cup P_{2}
$$

is a homotopy equivalence rel. $X_{1}$. Consider the following diagram where $j_{1}, j_{2}, k_{1}$, 
$k_{2}$ are inclusions. (Note that $i_{1}=k_{1} j_{1}$ and $i_{2}=k_{2} j_{2}$. )

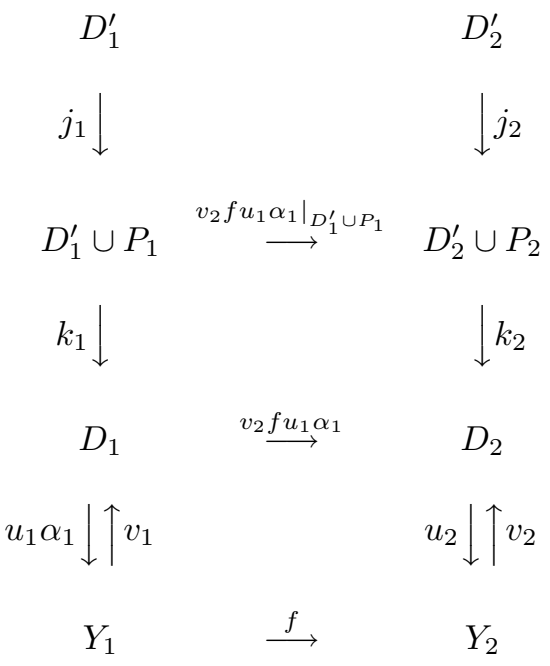

This gives rise to

$$
\begin{aligned}
& \tilde{K}_{0}\left(D_{1}^{\prime}\right) \quad \tilde{K}_{0}\left(D_{2}^{\prime}\right) \\
& j_{1 *} \downarrow \quad \downarrow j_{2 *} \\
& \tilde{K}_{0}\left(D_{1}^{\prime} \cup P_{1}\right) \stackrel{\left(\left.v_{2} f u_{1} \alpha_{1}\right|_{D_{1}^{\prime} \cup P_{1}}\right)_{*}}{\longrightarrow} \quad \tilde{K}_{0}\left(D_{2}^{\prime} \cup P_{2}\right) \\
& k_{1 *} \downarrow \quad \downarrow k_{2 *} \\
& \tilde{K}_{0}\left(D_{1}\right) \quad \stackrel{\left(v_{2} f u_{1} \alpha_{1}\right.}{\longrightarrow} * \quad \tilde{K}_{0}\left(D_{2}\right) \\
& \left(u_{1} \alpha_{1}\right)_{*} \downarrow \uparrow v_{1 *} \quad u_{2 *} \downarrow \uparrow v_{2 *} \\
& \tilde{K}_{0}\left(Y_{1}\right) \stackrel{f_{*}}{\longrightarrow} \quad \tilde{K}_{0}\left(Y_{2}\right)
\end{aligned}
$$

Each $P_{i}$ is compact, therefore $\sigma\left(P_{i}\right)=\sigma\left(D_{i}^{\prime} \cap P_{i}\right)=0$. Thus by the Sum Theorem [10] $\sigma\left(D_{i}^{\prime} \cup P_{i}\right)=j_{i *} \sigma\left(D_{i}^{\prime}\right)$. Since $\left.v_{2} f u_{1} \alpha_{1}\right|_{D_{1}^{\prime} \cup P_{1}}$ is a homotopy equivalence $\left(\left.v_{2} f u_{1} \alpha_{1}\right|_{D_{1}^{\prime} \cup P_{1}}\right)_{*} \sigma\left(D_{1}^{\prime} \cup P_{1}\right)=\sigma\left(D_{2}^{\prime} \cup P_{2}\right)$ which gives us:

$$
\begin{gathered}
\left(\left.v_{2} f u_{1} \alpha_{1}\right|_{D_{1}^{\prime} \cup P_{1}}\right)_{*} j_{1 *} \sigma\left(D_{1}^{\prime}\right)=j_{2 *} \sigma\left(D_{2}^{\prime}\right) \\
k_{2 *}\left(\left.v_{2} f u_{1} \alpha_{1}\right|_{D_{1}^{\prime} \cup P_{1}}\right)_{*} j_{1 *} \sigma\left(D_{1}^{\prime}\right)=k_{2 *} j_{2 *} \sigma\left(D_{2}^{\prime}\right)=i_{2 *} \sigma\left(D_{2}^{\prime}\right) \\
\left(v_{2} f u_{1} \alpha_{1}\right)_{*} k_{1 *} j_{1 *} \sigma\left(D_{1}^{\prime}\right)=i_{2 *} \sigma\left(D_{2}^{\prime}\right) \\
v_{2 *} f_{*} u_{1 *} i_{1 *} \sigma\left(D_{1}^{\prime}\right)=i_{2 *} \sigma\left(D_{2}^{\prime}\right) \\
u_{2 *} v_{2 *} f_{*} u_{1 *} i_{1 *} \sigma\left(D_{1}^{\prime}\right)=u_{2 *} i_{2 *} \sigma\left(D_{2}^{\prime}\right) \\
f_{*} u_{1 *} i_{1 *} \sigma\left(D_{1}^{\prime}\right)=u_{2 *} i_{2 *} \sigma\left(D_{2}^{\prime}\right) .
\end{gathered}
$$


The following corollary shows that $\sigma(Y, X)$ is well defined by demonstrating that $\sigma(Y, X)$ is independent of the choice of $D^{\prime}$.

Corollary 4.7. Suppose $(Y, X)$ is a finitely dominated $C W$ pair stable with respect to $\left(D^{\prime}, u, v\right)$ and $\left(D_{1}^{\prime}, u_{1}, v_{1}\right)$. Then $u_{*} i_{*} \sigma\left(D^{\prime}\right)=u_{1 *} i_{1 *} \sigma\left(D_{1}^{\prime}\right)$.

Proof. In the above proof let $Y_{1}=Y_{2}=Y$ and let $f=i d$.

Corollary 4.8 (Weak Invariance). Let $\left(Y_{1}, X_{1}\right)$ and $\left(Y_{2}, X_{2}\right)$ be finitely dominated $C W$ pairs. Suppose there exists a homotopy equivalence $f: Y_{1} \rightarrow Y_{2}$ such that

$$
\left.f\right|_{X_{1}}: X_{1} \rightarrow X_{2}
$$

is a $C W$ isomorphism. Then $f_{*} \sigma\left(Y_{1}, X_{1}\right)=\sigma\left(Y_{2}, X_{2}\right)$.

Lemma 4.9. Let $\left(Z, Z_{0}\right)$ be a $C W$ pair such that $Z_{0}$ is finite. Suppose $Z$ is homotopy equivalent to some finite $C W$ complex $K$. Then there exists a finite $C W$ complex $P$ such that $Z_{0} \subset P$ and there is a homotopy equivalence $P \rightarrow Z$ rel. $Z_{0}$.

Proof. There exist maps $f: Z \rightarrow K, g: K \rightarrow Z$ such that $g f \simeq i d$ and $f g \simeq i d$. Let $P=M\left(\left.f\right|_{Z_{0}}\right)$. Consider maps

$$
P \stackrel{r}{\longrightarrow} K \stackrel{g}{\longrightarrow} Z
$$

where $r$ is the collapse to the base along the rays of the mapping cylinder. Since $g$ and $r$ are both homotopy equivalences, $g r$ is also a homotopy equivalence.

Observe that $\left.g r\right|_{Z_{0}}=\left.g f\right|_{Z_{0}} \simeq i d_{Z_{0}}$. By the Homotopy Extension Theorem $g r$ is homotopic to some $g^{\prime}$ such that $\left.g^{\prime}\right|_{Z_{0}}=i d_{Z_{0}} .\left.g^{\prime}\right|_{Z_{0}}: Z_{0} \rightarrow Z_{0}$ is a homeomorphism. Thus, by Proposition 3.2 of [3], there exists $f^{\prime}$ such that $f^{\prime} g^{\prime} \simeq i d$ rel. $Z_{0}$ and $g^{\prime} f^{\prime} \simeq i d$ rel. $Z_{0}$.

Theorem 4.10 (Fundamental Property of the Relative Finiteness Obstruction). Let $(Y, X)$ be a finitely dominated $C W$ pair. Then $\sigma(Y, X)=0$ if and only if $X \hookrightarrow Y$ can be extended to a homotopy equivalence $X \cup P \rightarrow Y$ rel. $X$ where $P$ is a finite $C W$ complex such that $P \cap X$ is a subcomplex of both $X$ and $P$.

Proof. Suppose $X \hookrightarrow Y$ can be extended to a homotopy equivalence $u: X \cup P \rightarrow$ $Y$. Since $P$ is a finite $\mathrm{CW}$ complex, $(Y, X)$ is stable with respect to $(P, u)$. Thus $\sigma(Y, X)=u_{*} i_{*} \sigma(P)$. But $P$ is finite, therefore $\sigma(P)=0$ and $\sigma(Y, X)=0$.

Conversely, assume $\sigma(Y, X)=0$. Suppose that $(Y, X)$ is stable with respect to $\left(D^{\prime}, u, v\right)$. Then we have:

$$
\begin{gathered}
u_{*} i_{*} \sigma\left(D^{\prime}\right)=0 \\
v_{*} u_{*} i_{*} \sigma\left(D^{\prime}\right)=0 \\
(v u)_{*} i_{*} \sigma\left(D^{\prime}\right)=0 \\
i_{*} \sigma\left(D^{\prime}\right)=0 \in \tilde{K}_{0}(D) .
\end{gathered}
$$

Since $D^{\prime}$ is finitely dominated, $\sigma\left(D^{\prime}\right) \in \tilde{K}_{0}\left(D^{\prime}\right)$. There exists a compact subset $A$ of $D^{\prime}$, an inclusion map $j: A \hookrightarrow D^{\prime}$ and $\tau \in \tilde{K}_{0}(A)$ such that $\sigma\left(D^{\prime}\right)=j_{*}(\tau)$. Thus $i_{*} \sigma\left(D^{\prime}\right)=i_{*} j_{*}(\tau)=0 \in \tilde{K}_{0}(D)$.

There exists a compact set $B$ such that $A \subset B \subset D$ and an inclusion map $\alpha: A \hookrightarrow$ $B$ such that $\alpha_{*}(\tau)=0$. Without loss of generality we may assume that $X \cap D^{\prime} \subset B$. 
By the Sum Theorem

$$
\sigma\left(D^{\prime} \cup B\right)=\omega_{1 *} \sigma\left(D^{\prime}\right)+\omega_{2 *} \sigma(B)-\omega_{3 *} \sigma\left(D^{\prime} \cap B\right),
$$

where $\omega_{1}, \omega_{2}$ and $\omega_{3}$ are appropriate inclusions. But $B$ and $D^{\prime} \cap B$ are compact therefore $\omega_{2 *} \sigma(B)=\omega_{3 *} \sigma\left(D^{\prime} \cap B\right)=0$.

To show that $\omega_{1 *} \sigma\left(D^{\prime}\right)=0$ recall that $\sigma\left(D^{\prime}\right)=j_{*}(\tau)$. Observe that $\omega_{1} j=\beta \alpha$, where $\beta: B \hookrightarrow D^{\prime} \cup B$ is an inclusion. Thus,

$$
\beta_{*} \alpha_{*}(\tau)=\omega_{1 *} j_{*}(\tau)
$$

but $\alpha_{*}(\tau)=0$; therefore,

$$
\omega_{1 *} \sigma\left(D^{\prime}\right)=\omega_{1 *} j_{*}(\tau)=\beta_{*} \alpha_{*}(\tau)=0 .
$$

Thus, $\sigma\left(D^{\prime} \cup B\right)=0$ and $D^{\prime} \cup B$ is homotopy equivalent to some finite CW complex $K$.

Let $Z=D^{\prime} \cup B$ and $Z_{0}=\left(D^{\prime} \cup B\right) \cap X$. By Lemma 4.9 there exists a finite CW complex $P$ such that $Z_{0} \subset P$, and there is a homotopy equivalence $P \rightarrow Z$ rel. $Z_{0}$. We can extend this homotopy equivalence to the rest of $X$ by the identity to obtain a homotopy equivalence $P \cup X \rightarrow Z \cup X=D$ rel. $X$. But $D \simeq Y$ rel. $X$. Therefore $P \cup X \simeq Y$ rel. $X$.

Theorem 4.11. Let $(Y, X)$ be a $C W$ pair. Suppose $Y$ is finitely dominated rel. $X$, and $X$ is finitely dominated, then $Y$ is finitely dominated and

$$
\sigma(Y)=\sigma(Y, X)+i_{*} \sigma(X)
$$

where $i: X \hookrightarrow Y$ is an inclusion map.

Proof. There exists a finite subcomplex $C$ of $Y$ such that the inclusion $i: X \cup C \hookrightarrow$ $Y$ is a homotopy domination. $C$ and $X \cap C$ are finite therefore they are finitely dominated. By the Sum Theorem $X \cup C$ must be finitely dominated. Thus there exists a finite $\mathrm{CW}$ complex $P$ such that $P \stackrel{f}{\rightarrow} X \cup C$ is a homotopy domination. But then if $P \rightarrow Y$ is also a homotopy domination.

$\sigma(Y, X)=u_{*} j_{*} \sigma\left(D^{\prime}\right)$ where $D^{\prime}$ and $u$ are such that $(Y, X)$ is stable with respect to $\left(D^{\prime}, u\right)$, and $j: D^{\prime} \hookrightarrow D^{\prime} \cup X$ is an inclusion. It follows from the Sum Theorem that $D^{\prime} \cup X$ is finitely dominated and

$$
\sigma\left(D^{\prime} \cup X\right)=j_{*} \sigma\left(D^{\prime}\right)+k_{*} \sigma(X)-0,
$$

where $k: X \hookrightarrow D^{\prime} \cup X$ is an inclusion.

Applying $u_{*}$ to both sides we get

$$
u_{*} \sigma\left(D^{\prime} \cup X\right)=u_{*} j_{*} \sigma\left(D^{\prime}\right)+u_{*} k_{*} \sigma(X) .
$$

But $u$ is a homotopy equivalence therefore $u_{*} \sigma\left(D^{\prime} \cup X\right)=\sigma(Y)$. Since $u$ is the identity on $X$ we have $u_{*} k_{*} \sigma(X)=i_{*} \sigma(X)$ which yields the desired result.

Corollary 4.12. If $Y$ is finitely dominated with respect to $\emptyset$, then $Y$ is finitely dominated and $\sigma(Y, \emptyset)=\sigma(Y)$.

Lemma 4.13. Let $(Y, X)$ and $\left(Y^{\prime}, X^{\prime}\right)$ be $C W$ pairs. Suppose $Y^{\prime}$ is finitely dominated rel. $X^{\prime}$ and there exists a homotopy equivalence $f: Y^{\prime} \rightarrow Y$ such that $\left.f\right|_{X^{\prime}}: X^{\prime} \rightarrow X$ is a homeomorphism. Then $Y$ is finitely dominated rel. $X$. 
Proof. By Theorem 3.7 there exists a homotopy inverse $g: Y \rightarrow Y^{\prime}$ such that $g f \simeq$ $i d_{Y^{\prime}}$ rel. $X^{\prime}, f g \simeq i d_{Y}$ rel. $X$, and $\left.g\right|_{X}=\left.f^{-1}\right|_{X}$.

$Y^{\prime}$ is finitely dominated rel. $X^{\prime}$, therefore there exists a compact subcomplex $C^{\prime}$ of $Y^{\prime}$ such that the inclusion $i: X^{\prime} \cup C^{\prime} \hookrightarrow Y^{\prime}$ is a homotopy domination rel. $X^{\prime}$. Let $h$ be homotopy inverse of $i$. Then there exists a homotopy $H_{t}$ such that $H_{0}=i d_{Y^{\prime}}$, $H_{1}=i h$ and $\left.H_{t}\right|_{X^{\prime}}=i d$.

Define $G_{t}$ by

$$
G_{t}=f H_{t} g: Y \longrightarrow Y
$$

Then

$$
G_{0}=f g \simeq i d_{Y} \quad \text { rel. } X
$$

and

$$
G_{1}(Y)=f h g(Y) \subset f h\left(Y^{\prime}\right) \subset f\left(X^{\prime} \cup C^{\prime}\right)=X \cup f\left(C^{\prime}\right) .
$$

Theorem 4.14 (Relative Sum Theorem). Let $\left(Y_{1}, X_{1}\right),\left(Y_{2}, X_{2}\right)$ be finitely dominated $C W$ pairs. Let $Y=Y_{1} \cup Y_{2}, X=X_{1} \cup X_{2}, Y_{0}=Y_{1} \cap Y_{2}, X_{0}=X_{1} \cap X_{2}$. Suppose that $\left(Y_{0}, X_{0}\right)$ is finitely dominated. Then $(Y, X)$ is finitely dominated and

$$
\sigma(Y, X)=j_{1 *} \sigma\left(Y_{1}, X_{1}\right)+j_{2 *} \sigma\left(Y_{2}, X_{2}\right)-j_{0 *} \sigma\left(Y_{0}, X_{0}\right),
$$

where $j_{i}: Y_{i} \hookrightarrow Y(i=0,1,2)$ are inclusions.

Proof. Case 1. $X_{0}=X \cap Y_{0}$.

For $i=0,1,2$ we have $h_{t}^{i}: Y_{i} \rightarrow Y_{i}$ such that $h_{0}^{i}=i d_{Y_{i}}, h_{1}^{i}\left(Y_{i}\right) \subset C_{i} \cup X_{i}$ where each $C_{i}$ is compact in $Y_{i}$, and $\left.h_{t}^{i}\right|_{X_{i}}=i d_{X_{i}}$.

Using the Homotopy Extension Theorem we can extend $h_{t}^{0}$ to $k_{t}^{i}: Y_{i} \rightarrow Y_{i}(i=1,2)$ in such a way that $\left.k_{t}^{i}\right|_{X_{i}}=i d$.

Next we want to modify $h_{t}^{i}(i=1,2)$ so that $\left.h_{t}^{i}\right|_{X_{0} \cup C_{0}}=i d$. Observe that we already have $\left.h_{t}^{i}\right|_{X_{0}}=i d(i=1,2)$. We will perform the modification of $h_{t}^{1}$. Modification of $h_{t}^{2}$ is similar.

There exist finite subcomplexes $V$ and $K$ of $Y_{1}$ such that $C_{0} \subset \operatorname{Int}(V)$ and $V \subset \operatorname{Int}(K)$. Choose a map $\phi: K \backslash \operatorname{Int}(V) \rightarrow[0,1]$ such that $\phi(B d(K))=1$ and $\phi(B d(V))=0$.

Define

$$
\tilde{h}_{t}^{1}(y)= \begin{cases}h_{t}^{1}(y) & y \in Y_{1} \backslash \operatorname{Int}(K) \\ y & y \in V \\ h_{\phi(y) t}^{1}(y) & y \in K \backslash \operatorname{Int}(V) .\end{cases}
$$

Observe that $\tilde{h}_{0}^{1}=i d,\left.\tilde{h}_{t}^{1}\right|_{X_{1} \cup C_{0}}=i d$ and $\tilde{h}_{1}^{1}\left(Y_{1}\right) \subset P_{1} \cup X_{1}$ for some compact $P_{1} \subset$ $Y_{1}$. Similarly, we can modify $h_{t}^{2}$ so that $\tilde{h}_{0}^{2}=i d,\left.\tilde{h}_{t}^{2}\right|_{X_{2} \cup C_{0}}=i d$ and $\tilde{h}_{1}^{2}\left(Y_{2}\right) \subset P_{2} \cup X_{2}$ for some compact $P_{2} \subset Y_{2}$.

Define

$$
f_{t}^{i}(y)= \begin{cases}k_{2 t}^{i}(y) & 0 \leqslant t \leqslant 1 / 2 \\ \tilde{h}_{2 t-1}^{i} k_{1}^{i}(y) & 1 / 2 \leqslant t \leqslant 1 .\end{cases}
$$

Then $f^{i}$ is continuous and $f_{t}^{1}=f_{t}^{2}$ for all $y \in Y_{0}$. 
Let

$$
f_{t}(y)= \begin{cases}f_{t}^{1}(y) & y \in Y_{1} \\ f_{t}^{2}(y) & y \in Y_{2} .\end{cases}
$$

Observe that $f_{1}(Y) \subset\left(P_{1} \cup P_{2}\right) \cup X$ and the inclusion $\left(P_{1} \cup P_{2}\right) \cup X \hookrightarrow Y$ is a homotopy domination rel. $X$.

There exists a finite CW complex $M$ such that $P_{1} \cup P_{2} \subset M$. Then $M \cup X \hookrightarrow Y$ is a homotopy domination rel. $X$. Let $M_{i}=M \cap Y_{i}$, then $M_{i} \cup X_{i} \hookrightarrow Y_{i}$ is a homotopy domination rel. $X_{i}$ because $P_{i} \subset M_{i}$.

Using the mapping cylinder constructions we can define $D_{i}$ and $D_{i}^{\prime}(i=0,1,2)$ such that $\left(Y_{i}, X_{i}\right)$ is stable with respect to $\left(D_{i}^{\prime}, u_{i}\right)$ and $D_{1} \cap D_{2}=D_{0}$.

Recall that $u_{i}(y, t)=f_{t}^{i}(y)(i=0,1,2)$. Since $f_{t}^{1}=f_{t}^{2}$ for all $y \in Y_{0}, u_{1}$ agrees with $u_{2}$ on $Y_{0}$. Thus we can define $u$ by

$$
u(y)= \begin{cases}u_{1}(y) & y \in D_{1} \\ u_{2}(y) & y \in D_{2} .\end{cases}
$$

Then $u(y, t)=f_{t}(y)$. Let $D=D_{1} \cup D_{2}$ and $D^{\prime}=D_{1}^{\prime} \cup D_{2}^{\prime}$. Then $(Y, X)$ is stable with respect to $\left(D^{\prime}, u\right)$.

Let the following be inclusions:

$$
\begin{aligned}
& D_{i}^{\prime} \stackrel{\delta_{i}}{\hookrightarrow} D^{\prime} \stackrel{\theta}{\hookrightarrow} D \\
& D_{i}^{\prime} \stackrel{\alpha_{i}}{\hookrightarrow} D_{i} \stackrel{\beta_{i}}{\hookrightarrow} D .
\end{aligned}
$$

Then

$$
\begin{aligned}
\sigma\left(Y_{1} \cup Y_{2}, X_{1} \cup X_{2}\right) & =u_{*} \theta_{*} \sigma\left(D^{\prime}\right) \\
& =u_{*} \theta_{*} \delta_{1 *} \sigma\left(D_{1}^{\prime}\right)+u_{*} \theta_{*} \delta_{2 *} \sigma\left(D_{2}^{\prime}\right)-u_{*} \theta_{*} \delta_{0 *} \sigma\left(D_{0}^{\prime}\right) \\
& =u_{*} \beta_{1 *} \alpha_{1 *} \sigma\left(D_{1}^{\prime}\right)+u_{*} \beta_{2 *} \alpha_{2 *} \sigma\left(D_{2}^{\prime}\right)-u_{*} \beta_{0 *} \alpha_{0 *} \sigma\left(D_{0}^{\prime}\right) \\
& =j_{1 *}\left(\left.u\right|_{D_{1}}\right)_{*} \alpha_{1 *} \sigma\left(D_{1}^{\prime}\right)+j_{2 *}\left(\left.u\right|_{D_{2}}\right)_{*} \alpha_{2 *} \sigma\left(D_{2}^{\prime}\right) \\
& -j_{0 *}\left(\left.u\right|_{D_{0}}\right)_{*} \alpha_{0 *} \sigma\left(D_{0}^{\prime}\right) \\
& =j_{1 *} u_{1 *} \alpha_{1 *} \sigma\left(D_{1}^{\prime}\right)+j_{2 *} u_{2 *} \alpha_{2 *} \sigma\left(D_{2}^{\prime}\right)-j_{0 *} u_{0 *} \alpha_{0 *} \sigma\left(D_{0}^{\prime}\right) \\
& =j_{1 *} \sigma\left(Y_{1}, X_{1}\right)+j_{2 *} \sigma\left(Y_{2}, X_{2}\right)-j_{0 *} \sigma\left(Y_{0}, X_{0}\right) .
\end{aligned}
$$

Case 2. $X_{0} \neq X \cap Y_{0}$.

We will separate those parts of $X_{1}$ and $X_{2}$ which do not intersect in $Y_{0}$ and apply Case 1.

Consider $Y_{1} \times[0,1]$. We will identify $Y_{1}$ with $Y_{1} \times\{0\}$. Construct a reduced product $Y_{1} \times_{X_{0}}[0,1]$ by identifying $(x, t)$ with $(x, 0)$ for all $x \in X_{0}$ and $t \in[0,1]$. Observe that $Y_{1} \times X_{0}[0,1]$ is a CW complex and $X_{1} \times\{0\}, X_{1} \times\{1\}$ are subcomplexes. Clearly $Y_{1} \times_{X_{0}}[0,1]$ is finitely dominated rel. $X_{1} \times\{0\}$.

There exists a homeomorphism $f: Y_{1} \times_{X_{0}}[0,1] \rightarrow Y_{1} \times_{X_{0}}[0,1]$ such that $f(y, 0)=$ $(y, 1)$ for all $y \in Y_{1},\left.f\right|_{X_{1} \times\{0\}}: X_{1} \times\{0\} \rightarrow X_{1} \times\{1\}$ is a homeomorphism. Thus, by Lemma $4.13, Y_{1} \times_{X_{0}}[0,1]$ is finitely dominated rel. $X_{1} \times\{1\}$.

We can repeat this process for $Y_{2} \times_{X_{0}}[-1,0]$. Then $Y_{2} \times_{X_{0}}[-1,0]$ is finitely dominated rel. $X_{2} \times\{-1\}$. 
Let

$$
\begin{aligned}
Y_{1}^{\prime} & =Y_{1} \times_{X_{0}}[0,1] \\
Y_{2}^{\prime} & =Y_{2} \times_{X_{0}}[-1,0] \\
Y^{\prime} & =Y_{1}^{\prime} \cup Y_{2}^{\prime} \\
X_{1}^{\prime} & =X_{1} \times\{1\} \\
X_{2}^{\prime} & =X_{2} \times\{-1\} \\
X^{\prime} & =X_{1}^{\prime} \cup X_{2}^{\prime} .
\end{aligned}
$$

Observe that $Y_{1}^{\prime} \cap Y_{2}^{\prime}=Y_{0}$ and $X_{1}^{\prime} \cap X_{2}^{\prime}=X_{0}$. Since $Y_{i}^{\prime}$ is finitely dominated rel. $X_{i}^{\prime}(i=1,2)$ and $Y_{0}$ is finitely dominated rel. $X_{0}$, by Case 1 we know that $Y^{\prime}$ is finitely dominated rel. $X^{\prime}$.

Let $p: Y^{\prime} \rightarrow Y$ be the projection to the zero level. Then $p$ is a homotopy equivalence such that its restriction to $X^{\prime}$ is a homeomorphism. By Lemma 4.13, $Y$ is finitely dominated rel. $X$.

By Case 1,

$$
\sigma\left(Y^{\prime}, X^{\prime}\right)=k_{1 *} \sigma\left(Y_{1}^{\prime}, X_{1}^{\prime}\right)+k_{2 *} \sigma\left(Y_{2}^{\prime}, X_{2}^{\prime}\right)-k_{0 *} \sigma\left(Y_{0}^{\prime}, X_{0}^{\prime}\right) .
$$

Finally, by Weak Invariance

$$
\begin{aligned}
\sigma(Y, X) & =p_{*} \sigma\left(Y^{\prime}, X^{\prime}\right) \\
& =j_{1 *}\left(\left.p\right|_{Y_{1}^{\prime}}\right)_{*} \sigma\left(Y_{1}^{\prime}, X_{1}^{\prime}\right)+j_{2 *}\left(\left.p\right|_{Y_{2}^{\prime}}\right)_{*} \sigma\left(Y_{2}^{\prime}, X_{2}^{\prime}\right)-j_{0 *}\left(\left.p\right|_{Y_{0}^{\prime}}\right)_{*} \sigma\left(Y_{0}^{\prime}, X_{0}^{\prime}\right) \\
& =j_{1 *} \sigma\left(Y_{1}, X_{1}\right)+j_{2 *} \sigma\left(Y_{2}, X_{2}\right)-j_{0 *} \sigma\left(Y_{0}, X_{0}\right),
\end{aligned}
$$

where $k_{i}, j_{i}(i=0,1,2)$ are appropriate inclusions.

Theorem 4.15. Let $(Z, Y, X)$ be a $C W$ triple. Suppose $Z$ is finitely dominated rel. $Y$ and $Y$ is finitely dominated rel. $X$. Then $Z$ is finitely dominated rel. $X$ and

$$
\sigma(Z, X)=\sigma(Z, Y)+i_{*} \sigma(Y, X),
$$

where $i: Y \hookrightarrow Z$ is an inclusion map.

Proof. There exists a compact subcomplex $C$ of $Z$ such that the inclusion $i: Y \cup C \hookrightarrow$ $Z$ is a homotopy domination rel. $Y$. We are given that $Y$ is finitely dominated rel. $X$. $C$ is finite, therefore $C$ is finitely dominated. Thus, by the Relative Sum Theorem, $Y \cup C$ is finitely dominated rel. $X . X \subset Y$ therefore $Z$ is finitely dominated rel. $X$.

$\sigma(Z, Y)=u_{*} j_{*} \sigma\left(D^{\prime}\right)$ where $D^{\prime}$ and $u$ are such that $(Z, Y)$ is stable with respect to $\left(D^{\prime}, u\right)$ and $j: D^{\prime} \hookrightarrow D^{\prime} \cup Y$ is an inclusion.

$D^{\prime}$ is finitely dominated, therefore $D^{\prime}$ is finitely dominated rel. $\emptyset$. $Y$ is finitely dominated rel. $X . D^{\prime} \cap Y$ is compact. So it follows from the Relative Sum Theorem that $D^{\prime} \cup Y$ is finitely dominated rel. $\emptyset \cup X=X$ and

$$
\sigma\left(D^{\prime} \cup Y, X\right)=j_{*} \sigma\left(D^{\prime}\right)+k_{*} \sigma(Y, X)-0,
$$

where $k: Y \hookrightarrow D^{\prime} \cup Y$ is an inclusion. 
Applying $u_{*}$ to both sides we get

$$
u_{*} \sigma\left(D^{\prime} \cup Y, X\right)=u_{*} j_{*} \sigma\left(D^{\prime}\right)+u_{*} k_{*} \sigma(Y, X)
$$

But $u$ is a homotopy equivalence such that $\left.u\right|_{X}=i d$, therefore by Weak Invariance $u_{*} \sigma\left(D^{\prime} \cup Y, X\right)=\sigma(Z, X)$. Since $u$ is the identity on $Y$ we have $u_{*} k_{*} \sigma(Y, X)=$ $i_{*} \sigma(Y, X)$ which yields the desired result.

Corollary 4.16. Let $\left(Y_{1}, Y_{2}, \ldots, Y_{k}\right)$ be a $C W k$-tuple. Suppose that for $n=1, \ldots$, $k-1$ each $Y_{n}$ is finitely dominated rel. $Y_{n+1}$. Then $Y_{1}$ is finitely dominated rel. $Y_{k}$ and

$$
\sigma\left(Y_{1}, Y_{k}\right)=\sigma\left(Y_{1}, Y_{2}\right)+\sum_{n=2}^{k-1} i_{n *} \sigma\left(Y_{n}, Y_{n+1}\right)
$$

where $i_{n}: Y_{n} \hookrightarrow Y_{1}$ are inclusions.

Lemma 4.17. Suppose $(Y, X)$ and $(Z, X)$ are $C W$ pairs such that $Y \cap Z=X$ and $Y$ is finitely dominated rel. $X$, then $(Y \cup Z, Z)$ is finitely dominated and $\sigma(Y \cup Z, Z)=$ $i_{*} \sigma(Y, X)$ where $i: Y \hookrightarrow Y \cup Z$ is an inclusion.

Proof. $(Y \cup Z, Z)$ can be written as $(Y \cup Z, X \cup Z)$. Observe that $(Y, X),(Z, Z)$ and $(Y \cap Z, X \cap Z)$ are finitely dominated. Thus, by the Relative Sum Theorem, $(Y \cup Z, X \cup Z)$ is finitely dominated and we have:

$$
\begin{aligned}
\sigma(Y \cup Z, Z) & =\sigma(Y \cup Z, X \cup Z) \\
& =i_{*} \sigma(Y, X)+j_{*} \sigma(Z, Z)-k_{*} \sigma(Y \cap Z, X \cap Z) \\
& =i_{*} \sigma(Y, X)+0-0 \\
& =i_{*} \sigma(Y, X) .
\end{aligned}
$$

Lemma 4.18. Let $(Y, X)$ and $\left(Y_{1}, X_{1}\right)$ be $C W$ pairs such that $(Y, X)$ is finitely dominated. Suppose that $X$ is a subcomplex of $X_{1}$ and $i: X \hookrightarrow X_{1}$ is a homotopy equivalence rel. $X$. If $f: Y \rightarrow Y_{1}$ is a homotopy equivalence rel. $X$, then $\left(Y_{1}, X_{1}\right)$ is finitely dominated and $f_{*} \sigma(Y, X)=\sigma\left(Y_{1}, X_{1}\right)$.

Proof. Define $\tilde{f}: Y \cup X_{1} \rightarrow Y_{1}$ by

$$
\left.\tilde{f}\right|_{Y}=f,\left.\quad \tilde{f}\right|_{X_{1}}=i d
$$

then $\tilde{f}$ is a homotopy equivalence. By Theorem $3.7 \tilde{f}$ is a homotopy equivalence rel. $X_{1}$. By Lemma $4.17\left(Y \cup X_{1}, X_{1}\right)$ is finitely dominated. But $\tilde{f}$ is a homotopy equivalence such that $\left.\tilde{f}\right|_{X_{1}}: X_{1} \rightarrow X_{1}$ is a homeomorphism. Therefore by Lemma 4.13 $\left(Y_{1}, X_{1}\right)$ is finitely dominated.

By Lemma $4.17 \sigma\left(Y \cup X_{1}, X_{1}\right)=j_{*} \sigma(Y, X)$, where $j: Y \hookrightarrow Y \cup X_{1}$ is an inclusion. Since $\tilde{f}$ is a homotopy equivalence rel. $X_{1}$ and $\left.\tilde{f}\right|_{X_{1}}$ is a CW isomorphism, by Weak Invariance we have

$$
\tilde{f}_{*} \sigma\left(Y \cup X_{1}, X_{1}\right)=\sigma\left(Y_{1}, X_{1}\right)
$$

but

$$
\tilde{f}_{*} \sigma\left(Y \cup X_{1}, X_{1}\right)=\tilde{f}_{*} j_{*} \sigma(Y, X)=f_{*} \sigma(Y, X)
$$


Theorem 4.19 (Invariance). Let $(Y, X)$ and $\left(Y_{1}, X_{1}\right)$ be $C W$ pairs such that $(Y, X)$ is finitely dominated. Suppose $f: Y \rightarrow Y_{1}$ is a homotopy equivalence such that $\left.f\right|_{X}: X \rightarrow$ $X_{1}$ is a homotopy equivalence. Then $\left(Y_{1}, X_{1}\right)$ is finitely dominated and $f_{*} \sigma(Y, X)=$ $\sigma\left(Y_{1}, X_{1}\right)$.

Proof. $f: X \rightarrow X_{1}$ is a homotopy equivalence; therefore, $f: X \rightarrow X_{1} \times\{0\} \subset X \times$ $[0, \infty)$ is homotopic to a proper homotopy equivalence $f^{\prime}: X \rightarrow X_{1} \times[0, \infty)[4$, Lemma 21.1]. Let $M$ be the mapping cylinder of $\left.f^{\prime}\right|_{X}$. Let $F_{t}: X \rightarrow M$ be a homotopy such that $F_{0}=\left.f\right|_{X}$ and $F_{1}=i d_{X}$. Form $Y_{1} \cup M$ by attaching $M$ to $Y_{1}$ along $X_{1} \times\{0\}$. Use the Homotopy Extension Theorem to extend $F_{t}$ to $\tilde{F}_{t}: Y \rightarrow Y_{1} \cup M$ so that $\left.\tilde{F}_{t}\right|_{X}=F_{t}$ and $\tilde{F}_{0}=f$.

Let $g$ be a homotopy inverse of $f$. Let $c: Y_{1} \cup M \rightarrow Y_{1}$ be a map such that $\left.c\right|_{Y_{1}}=i d$ and $\left.c\right|_{M}$ is a collapse of $M$ to $X_{1} \times[0, \infty)$ followed by a collapse of $X_{1} \times[0, \infty)$ to $X_{1} \times\{0\}$. Then $\tilde{F}_{0}$ is a homotopy equivalence with a homotopy inverse $g c$. Since $\tilde{F}_{0} \simeq \tilde{F}_{1}, \tilde{F}_{1}$ is also a homotopy equivalence. Observe that $\left.\tilde{F}_{1}\right|_{X}=i d$, therefore by Theorem 3.7, $\tilde{F}_{1}$ is a homotopy equivalence rel. $X$. Also, $X \hookrightarrow M$ is a homotopy equivalence rel. $X$.

By Lemma $4.18\left(Y_{1} \cup M, M\right)$ is finitely dominated. Therefore there exists $h_{t}: Y_{1} \cup$ $M \rightarrow Y_{1} \cup M$ such that $h_{0}=i d,\left.h_{t}\right|_{M}=i d$ and $h_{1}\left(Y_{1} \cup M\right) \subset M \cup C$ for some compact subcomplex $C$. Consider

$$
H_{t}=\left.c h_{t}\right|_{Y_{1}}: Y_{1} \rightarrow Y_{1},
$$

$H_{0}=i d_{Y_{1}},\left.H_{t}\right|_{X_{1}}=i d$ and $H_{1}\left(Y_{1}\right) \subset X_{1} \cup c(C)$, where $c(C)$ is compact. It follows that $\left(Y_{1}, X_{1}\right)$ is finitely dominated.

Also by Lemma 4.18 we have

$$
\tilde{F}_{1 *} \sigma(Y, X)=\sigma\left(Y_{1} \cup M, M\right) .
$$

But by Lemma 4.17,

$$
\sigma\left(Y_{1} \cup M, M\right)=i_{*} \sigma\left(Y_{1}, X_{1}\right),
$$

where $i: Y_{1} \hookrightarrow Y_{1} \cup M$ is an inclusion. Thus, we have

$$
\begin{gathered}
\tilde{F}_{1 *} \sigma(Y, X)=i_{*} \sigma\left(Y_{1}, X_{1}\right) \\
c_{*} \tilde{F}_{1 *} \sigma(Y, X)=c_{*} i_{*} \sigma\left(Y_{1}, X_{1}\right),
\end{gathered}
$$

but $c \tilde{F}_{1} \simeq f$ because $c \tilde{F}_{1} \simeq c \tilde{F}_{0}=c f=f$, so

$$
f_{*} \sigma(Y, X)=\sigma\left(Y_{1}, X_{1}\right) .
$$

Theorem 4.20 (Relative Product Theorem). Let $\left(Y_{1}, X_{1}\right)$ and $\left(Y_{2}, X_{2}\right)$ be finitely dominated, path connected $C W$ pairs. Then

$$
\left(Y_{1} \times Y_{2},\left(Y_{1} \times X_{2}\right) \cup\left(X_{1} \times Y_{2}\right)\right)
$$

is finitely dominated and

$$
\begin{aligned}
\sigma\left(Y_{1} \times Y_{2},\left(Y_{1} \times X_{2}\right) \cup\left(X_{1} \times Y_{2}\right)\right) & =\sigma\left(Y_{1}, X_{1}\right) \cdot \sigma\left(Y_{2}, X_{2}\right)+ \\
& \chi\left(Y_{1}, X_{1}\right) j_{2 *} \sigma\left(Y_{2}, X_{2}\right)+\chi\left(Y_{2}, X_{2}\right) j_{1 *} \sigma\left(Y_{1}, X_{1}\right),
\end{aligned}
$$

where $j_{i}: Y_{i} \hookrightarrow Y_{1} \times Y_{2}(i=1,2)$ are inclusions. 
Proof. For $i=1,2$ suppose that $\left(Y_{i}, X_{i}\right)$ is stable with respect to $\left(D_{i}^{\prime}, u_{i}\right)$. Let $D_{i}=$ $D_{i}^{\prime} \cup X_{i}$. Let $X_{i}^{\prime}=X_{i} \cap D_{i}^{\prime}$. Note that by following the original constructions we can insure that each $X_{i}^{\prime}$ is compact.

(1) We will start by showing that $\left(D_{1} \times D_{2},\left(D_{1} \times X_{2}\right) \cup\left(X_{1} \times D_{2}\right)\right)$ is finitely dominated. The following figure will be helpful.

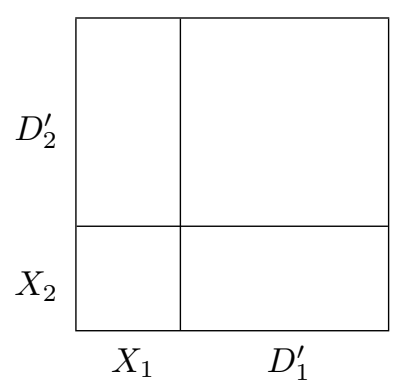

Figure 3: $D_{1} \times D_{2}$

Observe that

$$
D_{1} \times D_{2}=\left(D_{1}^{\prime} \times D_{2}^{\prime}\right) \cup\left[\left(X_{1} \times D_{2}\right) \cup\left(D_{1} \times X_{2}\right)\right] .
$$

Each $D_{i}^{\prime}$ is finitely dominated by the definition; thus,

$$
\left(D_{1}^{\prime} \times D_{2}^{\prime}, \emptyset\right)
$$

is finitely dominated.

Clearly

$$
\left(\left(X_{1} \times D_{2}\right) \cup\left(D_{1} \times X_{2}\right),\left(X_{1} \times D_{2}\right) \cup\left(D_{1} \times X_{2}\right)\right)
$$

is finitely dominated.

Finally we need to demonstrate that

$$
\left(\left(D_{1}^{\prime} \times D_{2}^{\prime}\right) \cap\left[\left(X_{1} \times D_{2}\right) \cup\left(D_{1} \times X_{2}\right)\right], \emptyset\right)
$$

is finitely dominated. But

$$
\left(D_{1}^{\prime} \times D_{2}^{\prime}\right) \cap\left[\left(X_{1} \times D_{2}\right) \cup\left(D_{1} \times X_{2}\right)\right]=\left(X_{1}^{\prime} \times D_{2}^{\prime}\right) \cup\left(D_{1}^{\prime} \times X_{2}^{\prime}\right) .
$$

This is finitely dominated by the Sum Theorem since each term of the union is finitely dominated by the Product Theorem [10] while the intersection is a compact set $X_{1}^{\prime} \times X_{2}^{\prime}$.

It is clear that

$$
u_{1} \times u_{2}: D_{1} \times D_{2} \rightarrow Y_{1} \times Y_{2}
$$

is a homotopy equivalence rel. $X_{1} \times X_{2}$. Thus, it restricts to a homotopy equivalence

$$
u_{1} \times\left. u_{2}\right|_{\left(D_{1} \times X_{2}\right) \cup\left(X_{1} \times D_{2}\right)}:\left(D_{1} \times X_{2}\right) \cup\left(X_{1} \times D_{2}\right) \rightarrow\left(Y_{1} \times X_{2}\right) \cup\left(X_{1} \times Y_{2}\right) .
$$

The desired result follows from Invariance. 
A RELATIVE VERSION OF THE FINITENESS OBSTRUCTION THEORY OF WALL 403

(2) We will first derive the formula for $\sigma\left(D_{1} \times D_{2},\left(D_{1} \times X_{2}\right) \cup\left(X_{1} \times D_{2}\right)\right)$. We will use inc to denote any appropriate inclusion.

$$
\begin{aligned}
& \sigma\left(D_{1} \times D_{2},\left(D_{1} \times X_{2}\right) \cup\left(X_{1} \times D_{2}\right)\right) \\
&=i n c_{*} \sigma\left(D_{1}^{\prime} \times D_{2}^{\prime}, \emptyset\right)+i n c_{*} \sigma\left(\left(X_{1} \times D_{2}\right) \cup\left(D_{1} \times X_{2}\right),\left(X_{1} \times D_{2}\right) \cup\left(D_{1} \times X_{2}\right)\right) \\
&-i n c_{*} \sigma\left(\left(X_{1}^{\prime} \times D_{2}^{\prime}\right) \cup\left(D_{1}^{\prime} \times X_{2}^{\prime}\right), \emptyset\right) \\
&= i n c_{*}\left(\sigma\left(D_{1}^{\prime}\right) \cdot \sigma\left(D_{2}^{\prime}\right)\right)+\chi\left(D_{1}^{\prime}\right) i n c_{*} \sigma\left(D_{2}^{\prime}\right)+\chi\left(D_{2}^{\prime}\right) i n c_{*} \sigma\left(D_{1}^{\prime}\right) \\
&-i n c_{*} \sigma\left(X_{1}^{\prime} \times D_{2}^{\prime}\right)-i n c_{*} \sigma\left(D_{1}^{\prime} \times X_{2}^{\prime}\right)+i n c_{*} \sigma\left(X_{1}^{\prime} \times X_{2}^{\prime}\right) \\
&= i n c_{*}\left(\sigma\left(D_{1}^{\prime}\right) \cdot \sigma\left(D_{2}^{\prime}\right)\right)+\chi\left(D_{1}^{\prime}\right) i n c_{*} \sigma\left(D_{2}^{\prime}\right)+\chi\left(D_{2}^{\prime}\right) i n c_{*} \sigma\left(D_{1}^{\prime}\right) \\
&-\chi\left(X_{1}^{\prime}\right) i n c_{*} \sigma\left(D_{2}^{\prime}\right)-\chi\left(X_{2}^{\prime}\right) i n c_{*} \sigma\left(D_{1}^{\prime}\right) \\
&= i n c_{*}\left(\sigma\left(D_{1}^{\prime}\right) \cdot \sigma\left(D_{2}^{\prime}\right)\right)+\left(\chi\left(D_{1}^{\prime}\right)-\chi\left(X_{1}^{\prime}\right)\right) i n c_{*} \sigma\left(D_{2}^{\prime}\right)+\left(\chi\left(D_{2}^{\prime}\right)\right. \\
&\left.-\chi\left(X_{2}^{\prime}\right)\right) i n c_{*} \sigma\left(D_{1}^{\prime}\right) \\
&= i n c_{*}\left(\sigma\left(D_{1}^{\prime}\right) \cdot \sigma\left(D_{2}^{\prime}\right)\right)+\chi\left(D_{1}^{\prime}, X_{1}^{\prime}\right) i n c_{*} \sigma\left(D_{2}^{\prime}\right)+\chi\left(D_{2}^{\prime}, X_{2}^{\prime}\right) i n c_{*} \sigma\left(D_{1}^{\prime}\right) \\
&= i n c_{*}\left(\sigma\left(D_{1}^{\prime}\right) \cdot \sigma\left(D_{2}^{\prime}\right)\right)+\chi\left(Y_{1}, X_{1}\right) i n c_{*} \sigma\left(D_{2}^{\prime}\right)+\chi\left(Y_{2}, X_{2}\right) i n c_{*} \sigma\left(D_{1}^{\prime}\right) .
\end{aligned}
$$

Applying $\left(u_{1} \times u_{2}\right)_{*}$ we get

$$
\begin{aligned}
& \sigma\left(Y_{1} \times Y_{2},\left(Y_{1} \times X_{2}\right) \cup\left(X_{1} \times Y_{2}\right)\right) \\
&=\left(u_{1} \times u_{2}\right)_{*} \sigma\left(D_{1} \times D_{2},\left(D_{1} \times X_{2}\right) \cup\left(X_{1} \times D_{2}\right)\right) \\
&=\left(u_{1} \times u_{2}\right)_{*} i n c_{*}\left(\sigma\left(D_{1}^{\prime}\right) \cdot \sigma\left(D_{2}^{\prime}\right)\right)+\chi\left(Y_{1}, X_{1}\right)\left(u_{1} \times u_{2}\right)_{*} i n c_{*} \sigma\left(D_{2}^{\prime}\right) \\
&+\chi\left(Y_{2}, X_{2}\right)\left(u_{1} \times u_{2}\right)_{*} i n c_{*} \sigma\left(D_{1}^{\prime}\right) \\
&= u_{1 *} i n c_{*} \sigma\left(D_{1}^{\prime}\right) \cdot u_{2 *} i n c_{*} \sigma\left(D_{2}^{\prime}\right)+\chi\left(Y_{1}, X_{1}\right) i n c_{*} u_{2 *} i n c_{*} \sigma\left(D_{2}^{\prime}\right) \\
&+\chi\left(Y_{2}, X_{2}\right) i n c_{*} u_{1 *} i c_{*} \sigma\left(D_{1}^{\prime}\right) \\
&= \sigma\left(Y_{1}, X_{1}\right) \cdot \sigma\left(Y_{2}, X_{2}\right)+\chi\left(Y_{1}, X_{1}\right) j_{2 *} \sigma\left(Y_{2}, X_{2}\right)+\chi\left(Y_{2}, X_{2}\right) j_{1 *} \sigma\left(Y_{1}, X_{1}\right)
\end{aligned}
$$

If in the above theorem we let $\left(Y_{2}, X_{2}\right)$ be $(K, \emptyset)$ where $K$ is (1) finite or (2) finitely dominated, then the result reduces to

$$
\begin{gathered}
\sigma\left(Y_{1} \times K, X_{1} \times K\right)=\chi(K) j_{1 *} \sigma\left(Y_{1}, X_{1}\right) \\
\sigma\left(Y_{1} \times K, X_{1} \times K\right)=\sigma\left(Y_{1}, X_{1}\right) \cdot \sigma(K)+\chi\left(Y_{1}, X_{1}\right) j_{2 *} \sigma(K) \\
+\chi(K) j_{1 *} \sigma\left(Y_{1}, X_{1}\right)
\end{gathered}
$$

It is interesting to note that if $\left(Y_{1}, X_{1}\right)$ and $\left(Y_{2}, X_{2}\right)$ are finitely dominated, $\left(Y_{1} \times\right.$ $\left.Y_{2}, X_{1} \times X_{2}\right)$ may not be finitely dominated. For example, let

$$
Z=\left\{(x, y) \mid(x-2 n)^{2}+y^{2}=1, n=0,1,2, \ldots\right\} .
$$

Clearly, $(Z, Z)$ is finitely dominated. Let $(Y, X)$ be a finitely dominated pair satisfying the following condition: For any homotopy domination $C \cup X \hookrightarrow Y, X$ is not a strong deformation retract of $C \cup X$. Then $(Z \times Y, Z \times X)$ is not finitely dominated.

\section{References}

[1] H. Bass, A. Heller, and R. Swan, The Whitehead group of a polynomial extension, Inst. Hautes Études Sci. Publ. Math. (1964), No. 22, 61-79. 
[2] C. Borsuk, Theory of retracts, Monografie Matematyczne, Tom 44 Państwowe Wydawnictwo Naukowe, Warsaw, 1967.

[3] T. A. Chapman, Homotopy conditions which detect simple homotopy equivalences, Pacific J. Math., 80 (1979), No. 1, 13-46.

[4] T. A. Chapman, Lectures on Hilbert cube manifolds, Regional Conference Series in Mathematics, No. 28, American Mathematical Society, Providence, R. I., 1976.

[5] T. A. Chapman, Controlled simple homotopy theory and applications, Lecture Notes in Mathematics, 1009. Springer-Verlag, Berlin, 1983.

[6] M. M. Cohen, A course in simple homotopy theory, Springer-Verlag, New YorkBerlin, 1973.

[7] S. Ferry, A simple-homotopy approach to finiteness obstruction, Lecture Notes in Mathematics, 870, Springer-Verlag, Berlin-New York, 1981.

[8] M. Greenberg, Lectures on algebraic topology, W. A. Benjamin, Inc., New YorkAmsterdam, 1967.

[9] H. Schubert, Topology, Allyn and Bacon, Boston, 1968.

[10] L. C. Siebenmann, The obstruction to finding a boundary for an open manifold of dimension greater than five, Princeton Thesis, 1965.

[11] L. C. Siebenmann, Infinite simple homotopy types, Nederl. Akad. Wetensch. Proc. Ser. A 73 No. 5 and Indag. Math. 32 (1970), 479-495.

[12] K. Varadarajan, The finiteness obstruction of C. T. C. Wall, J. Wiley and Sons, New York, 1989.

[13] C. T. C. Wall, Finiteness conditions for CW complexes, Annals of Math. (2), 81 (1965), 56-69.

[14] C. T. C. Wall, Finiteness conditions for CW complexes II, Proc. Roy. Soc. Ser. A, 295 (1966), 129-139.

Anna Davis davisa@ohiodominican.edu

Mathematics Department, Ohio Dominican University, 1216 Sunbury Rd., Columbus, OH, 43219, USA 